\title{
The Increased Risk of Death from Ischaemic Heart Disease in First Degree Relatives of I2I Men and 96 Women with Ischaemic Heart Disease
}

\author{
J. SLACK and $\mathrm{K}$. A. EVANS \\ From the Medical Research Council, Clinical Genetics Research Unit, Institute of Child Health, London W.C.I
}

The risks of death from ischaemic heart disease, cerebral haemorrhage, and thrombosis, and the risks of death from all causes have been estimated among the first degree relatives of male and female index patients with ischaemic heart disease. These risks have been compared with the figures published by the Registrar General for England and Wales and with the risks to relatives of a control group. It is considered that the Registrar General's figures provide the best comparison for mortality, but the control series provides useful comparison for morbidity.

There has been much speculation but little concrete evidence about the hereditary aspects of ischaemic heart disease. Gertler and White (1954) studied family histories of 97 male patients with coronary heart disease before 4I years, excluding hypertensive patients, and found an incidence of coronary heart disease which was twice as high amongst fathers of patients compared with controls, but less increase amongst mothers compared with their control sample. Thomas and Cohen (1955) studied family histories of 266 consecutive medical students at Johns Hopkins University and found a 4-fold increase amongst brothers of male patients. In an extensive twin study, Harvald and Hauge (1963) found no difference in concordance rates for the occurrence of coronary occlusion between monozygotic and dizygotic twins of like sex, whereas dizygotic twins of unlike sex showed a significant difference. Rose (1964) has more recently reported on family histories of 100 patients with ischaemic heart disease and found a 3-fold increase in mortality from ischaemic heart disease amongst parents of male patients and a higher mortality at all ages from unrelated illnesses. In these series there have been few female patients studied, and in some there has been a lack of documentary evidence

Received August 8, 1966. of the causes of mortality of the relatives, and it seems that the lack of evidence still prohibits definite conclusions as to risks to near relatives of patients with ischaemic heart disease.

We have collected pedigrees of nearly equal numbers of men and women with ischaemic heart disease and attempted to obtain documents of confirmation of every cause of death or serious morbidity in first degree relatives.

\section{Subjects and Methods}

Index Patients. The patients were $12 \mathrm{I}$ men and 96 women who had attended hospital with ischaemic heart disease; this was the only criterion for selection for the series. Diagnosis of ischaemic heart disease was made in each case on clinical evidence supported by ECG changes compatible with ischaemic heart disease. Male patients were all less than 60 years old and female patierts less than 70 at the onset of ischaemic heart disease. The patients were drawn from hospitals serving the central and north London areas and are shown in Table IA. Collection of the series of male patients continued over a period of 7 months at the Central Middlesex Hospital and over a further period of 8 months at the National Heart Hospital. Collection of women patients took longer and was carried out for 7 months at the Central Middlesex Hospital and a further 15 months at the National Heart Hospital and other hospitals in order to collect sufficient numbers for the series.

Controls. The group consisted of ro4 men and I05 women. 95 men and 72 women were employees from the London Office of the Prudential Assurance Company; 3 men and I woman were hospital patients, 29 controls were wives of the patients, and 6 men and 3 women were medical colleagues (see Table IB). All were within the same age range as the patients. None was aware of having ischaemic heart disease.

Family Histories. Pedigrees were drawn up to include every first degree relative of patients and controls. Permission to verify causes of death was requested 
TABLE IA

WHOLE SAMPLE OF INDEX PATIENTS SHOWING THEIR SOURCES AND REASONS FOR EXCLUSION

\begin{tabular}{|c|c|c|c|c|c|}
\hline Patients & $\begin{array}{l}\text { National } \\
\text { Heart } \\
\text { Hospital }\end{array}$ & $\begin{array}{c}\text { Central } \\
\text { Middlesex } \\
\text { Hospital }\end{array}$ & $\begin{array}{l}\text { The } \\
\text { Middlesex } \\
\text { Hospital }\end{array}$ & $\begin{array}{l}\text { Elizabeth } \\
\text { Garrett } \\
\text { Anderson } \\
\text { Hospital }\end{array}$ & Total \\
\hline $\begin{array}{l}\text { Male patients } \\
\text { less } \\
\text { (a) non-co-operators } \\
\text { (b) foreign, etc. }\end{array}$ & $\begin{array}{r}45 \\
-4\end{array}$ & $\begin{array}{r}88 \\
6 \\
6\end{array}$ & $\begin{array}{l}7 \\
\mathbf{I} \\
\mathbf{2}\end{array}$ & $\begin{array}{l}- \\
-\end{array}$ & $\begin{array}{r}140 \\
7 \\
12\end{array}$ \\
\hline No. in study & $4 I$ & 76 & 4 & - & 121 \\
\hline $\begin{array}{l}\text { Female patients } \\
\text { less } \\
\text { (a) non-co-operators } \\
\text { (b) foreign, etc. }\end{array}$ & $\begin{array}{r}73 \\
-2\end{array}$ & $\begin{array}{r}29 \\
6 \\
1\end{array}$ & $\begin{array}{l}\mathbf{I} \\
- \\
-\end{array}$ & $\begin{array}{r}2 \\
-\end{array}$ & $\begin{array}{r}105 \\
6 \\
3\end{array}$ \\
\hline No. in study & 71 & 22 & $\mathbf{I}$ & 2 & 96 \\
\hline
\end{tabular}

TABLE IB

WHOLE SAMPLE OF CONTROLS, SHOWING THEIR SOURCES AND REASONS FOR EXCLUSION

\begin{tabular}{|c|c|c|c|c|c|}
\hline Controls & Prudential & $\begin{array}{l}\text { Hospital } \\
\text { Controls }\end{array}$ & Spouses & Colleagues & Total \\
\hline $\begin{array}{l}\text { Male controls } \\
\text { less } \\
\text { (a) non-co-operators } \\
\text { (b) foreign, etc. }\end{array}$ & $\begin{array}{l}95 \\
-\end{array}$ & $\begin{array}{l}5 \\
I \\
I\end{array}$ & $\begin{array}{l}- \\
-\end{array}$ & $\begin{array}{r}6 \\
-\end{array}$ & $\begin{array}{r}106 \\
\text { I } \\
\text { I }\end{array}$ \\
\hline No. in study & 95 & 3 & - & 6 & 104 \\
\hline $\begin{array}{l}\text { Female controls } \\
\text { less } \\
\text { (a) non-co-operators } \\
\text { (b) foreign, etc. }\end{array}$ & $\begin{array}{r}73 \\
\mathbf{I} \\
\end{array}$ & $\begin{array}{r}\mathbf{1} \\
- \\
-\end{array}$ & $\begin{array}{r}32 \\
2 \\
1\end{array}$ & $\begin{array}{l}4 \\
-1\end{array}$ & $\begin{array}{r}110 \\
3 \\
2 \\
\end{array}$ \\
\hline No. in study & 72 & $\mathbf{I}$ & 29 & 3 & 105 \\
\hline
\end{tabular}

from each person entering the study and if this was withheld or if several relatives were known to be untraceable the subject was excluded from the study. There were 7 male and 6 female patients and I male and 3 female controls who were unwilling for us to document causes of death in their relatives. There were 12 male and 3 female patients, and 1 male and 2 female controls who were excluded because the majority of their relatives had died in Eastern Europe or in war-time Germany, and documentary evidence of the causes of death could not be obtained. The cause of death of each relative over I 5 years was recorded and verified by death certificates (with the exception of deaths in action during the two Great Wars) and where possible all causes of serious morbidity were confirmed by hospital records or from family doctors. Only limited attempts were made to trace deaths over 80 years and these deaths were not claimed as coronary artery disease unless death certificates were obtained. Table II showsీ the total numbers of relatives and the success and failure in obtaining confirmatory evidence of death and the reasons for failure.

The number of death certificates obtained was 699 , and of these the 397 with any mention of heart disease or cerebral catastrophe were sent to the General Register Office where they were classified by the criteria in use at the time of issue of the certificate. We are much indebted to the Registrap General for this service, because there is no doubtD discrepancies would have occurred if we had at tempted to classify the certificates ourselves by reference to the headings in the Registrar General' Statistical Review. 
TABLE II

NUMBERS OF RELATIVES OF INDEX PATIENTS AND CONTROLS AND DETAILS OF DOCUMENTS CONFIRMING DEATHS

\begin{tabular}{|c|c|c|c|c|c|c|c|c|c|c|}
\hline & & \multirow{3}{*}{ Total } & \multicolumn{4}{|c|}{ Relatives } & \multicolumn{4}{|c|}{ Deaths } \\
\hline & & & \multirow{2}{*}{$\begin{array}{l}\text { Not on Tables; } \\
\text { Insufficient } \\
\text { Evidence of } \\
\text { Cause of Death }\end{array}$} & \multicolumn{3}{|c|}{ On Tables } & \multirow{2}{*}{$\begin{array}{c}\text { Documented } \\
\begin{array}{c}\text { Death } \\
\text { Certificates }\end{array}\end{array}$} & \multicolumn{3}{|c|}{ Not Documented } \\
\hline & & & & Total & Alive & Dead & & War & $\begin{array}{l}\text { Over } \\
80 \text { years }\end{array}$ & Others \\
\hline $\begin{array}{l}\text { Male } \\
\text { patients } \\
\text { I } 2 \text { I }\end{array}$ & $\begin{array}{l}\text { Fathers } \\
\text { Brothers } \\
\text { Mothers } \\
\text { Sisters } \\
\end{array}$ & $\begin{array}{l}121 \\
198 \\
121 \\
188\end{array}$ & $\begin{array}{l}6 \\
1 \\
3 \\
1\end{array}$ & $\begin{array}{l}115 \\
197 \\
118 \\
187\end{array}$ & $\begin{array}{r}11 \\
156 \\
28 \\
171\end{array}$ & $\begin{array}{r}104 \\
41 \\
90 \\
16\end{array}$ & $\begin{array}{l}89 \\
33 \\
77 \\
12\end{array}$ & $\begin{array}{l}5 \\
6 \\
1 \\
0\end{array}$ & $\begin{array}{l}3 \\
0 \\
4 \\
0\end{array}$ & $\begin{array}{l}7 \\
2 \\
8 \\
4\end{array}$ \\
\hline $\begin{array}{l}\text { Female } \\
\text { patients } \\
96\end{array}$ & $\begin{array}{l}\text { Fathers } \\
\text { Brothers } \\
\text { Mothers } \\
\text { Sisters }\end{array}$ & $\begin{array}{r}96 \\
166 \\
96 \\
144\end{array}$ & $\begin{array}{l}6 \\
1 \\
3 \\
2\end{array}$ & $\begin{array}{r}90 \\
165 \\
93 \\
142\end{array}$ & $\begin{array}{r}3 \\
118 \\
15 \\
115\end{array}$ & $\begin{array}{l}87 \\
47 \\
78 \\
27\end{array}$ & $\begin{array}{l}83 \\
41 \\
71 \\
24\end{array}$ & $\begin{array}{l}2 \\
4 \\
0 \\
2\end{array}$ & $\begin{array}{l}1 \\
0 \\
5 \\
0\end{array}$ & $\begin{array}{l}\mathbf{I} \\
\mathbf{2} \\
\mathbf{2} \\
\mathbf{I}\end{array}$ \\
\hline $\begin{array}{l}\text { Female } \\
\text { controls } \\
\text { ros }\end{array}$ & $\begin{array}{l}\text { Fathers } \\
\text { Brothers } \\
\text { Mothers } \\
\text { Sisters }\end{array}$ & $\begin{array}{l}105 \\
112 \\
105 \\
141\end{array}$ & $\begin{array}{l}3 \\
0 \\
1 \\
0\end{array}$ & $\begin{array}{l}102 \\
112 \\
104 \\
141\end{array}$ & $\begin{array}{r}27 \\
91 \\
50 \\
133\end{array}$ & $\begin{array}{l}75 \\
21 \\
54 \\
8\end{array}$ & $\begin{array}{l}69 \\
13 \\
50 \\
8\end{array}$ & $\begin{array}{l}2 \\
8 \\
0 \\
0\end{array}$ & $\begin{array}{l}2 \\
0 \\
2 \\
0\end{array}$ & $\begin{array}{l}2 \\
0 \\
2 \\
0\end{array}$ \\
\hline
\end{tabular}

Tables showing years 'at risk' were constructed for every 5 calendar year period from 1930, and divided into Io-year age-groups from 20 years. Relatives were considered in 4 groups, fathers and adult brothers, mothers and adult sisters of male and female patients. Patients were divided into groups by their age at the onset of ischaemic heart disease and separate tables were prepared for each group. Parents were entered on the table at their age at the birth of the index patient, which was the earliest time at which they could be considered to be 'at risk' for purposes of the study. Brothers and sisters were entered at 20 years. Entries were in 5 calendar year groups by date of birth and were scored as entering midway through the appropriate ro-year age period. Deceased relatives left the table midway through the appropriate ro-year age period, and in each case the Registrar General's classification of the cause of death was recorded. Living relatives left the table in 1965 and were scored as leaving halfway through the appropriate ro-year period. Those who reached 75 years and over were allowed a life expectancy corresponding to that given in the National Life Tables instead of a Io-year span. (1930-I945 life expectancy for men 6.4 years and for women $7.4,1946-1955$ for men 6.7 years and for women 8.1, 1956 to 1964 for men 7.I years and for women 8.8.) In this way it was possible to calculate the number of man years and the calendar years during which the relatives were 'at risk' of dying. Similar tables were constructed for the relatives of male and female controls.

Before 1930, classification and diagnosis of ischaemic heart disease were not sufficiently precise for this study, and, therefore, no use was made, in our calculations, of certificates issued before 1930, and the life experience of relatives before 1930 was excluded. However, details of death before 1930 are given in Appendix II.

The Registrar General's tables were used to calculate the risks of dying from all causes, from ischaemic heart disease, and from cerebrovascular disorders, at 5-yearly intervals from 1930 to the present. The Registrar General's categories used can be seen in Appendix I. Between 1930 and the present day death certificates with special mention of coronary artery disease have been distinguished from other arteriosclerotic heart disease in categories 420 and 94. These categories have been mainly used in this study. For each 5-year period the risks of death in the general population in the categories mentioned were calculated for age-groups 20-34, $35-44,45-54,55-64,65-74$, and 75 and over. From the tables then, the expected number of deaths amongst relatives was calculated in each category and at each age-group. The observed number of deaths was compared with the expected number of deaths in each age-group and expressed as a ratio. Tables of years 'at risk' were constructed for controls in the same way. There was no grouping of 
TABLE III

RISKS TO MEN AND WOMEN OF DYING FROM ISCHAEMIC HEART DISEASE (REGISTRAR GENERAL'S CATEGORY 420) IN 1962 IN IO-YEAR PERIODS

\begin{tabular}{c|c|c}
\hline Age (yr.) & Men & Women \\
\hline $35-44$ & 0.00541 & 0.00072 \\
$45-54$ & 0.02131 & 0.00358 \\
$55-64$ & 0.06391 & 0.01720 \\
$65-74$ & 0.13946 & 0.06126 \\
75 to expected age at \\
death (see text)
\end{tabular}

controls by age, but the life experience of their relatives was considered in the same age-groups.

Risks calculated from the Registrar General's tables for 1962 and set out in Table III show the risks of dying in category 420 , encountered by men and women at ro-year age-groups from 35 years. This shows that the risks of dying from ischaemic heart disease in men are similar to those of women who are ro years older. The risk of dying in cate gory 420 for men becomes about 35/10,000 per year by age 55 years and for women by about 65 years.+ We have, therefore, divided the male patients and relatives into a 'younger' and 'older' age-group a市 55 years and the women patients and relatives at 65 years.

\section{Results}

Table IV shows the number of years 'at risk' in $\vec{\circ}$ each age-group contributed by male and female $\vec{\omega}$

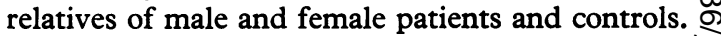

From the years 'at risk' and from the risks of death in the general population in that age-group $p_{\omega}$ and in the appropriate secular period, the number $\triangle$ of deaths expected amongst relatives of patients is compared with the number observed.

Risks of Death from Ischaemic Heart Disease in Patients' Relatives. Tables VA and VB show for male and female patients respectively, the number of deaths observed $(\mathrm{O})$ and the expected number 3 of deaths (E) in relatives calculated from the yearso 'at risk' and the risks from the Registrar General's

TABLE IV

YEARS AT RISK OF RELATIVES OF INDEX PATIENTS (DISTINGUISHED BY AGE OF ONSET) AND CONTROLS

\begin{tabular}{|c|c|c|c|c|c|c|c|}
\hline \multirow{3}{*}{$\begin{array}{l}\text { Age of } \\
\text { Relative }\end{array}$} & \multicolumn{5}{|c|}{ Patients' Ages at Onset of Ischaemic Heart Disease } & \multirow{3}{*}{ Total } & \multirow{3}{*}{$\begin{array}{l}\text { Male Controls } \\
\text { (all ages) }\end{array}$} \\
\hline & \multicolumn{5}{|c|}{ Male Patients } & & \\
\hline & $35-44$ & $45-49$ & $50-54$ & $55-59$ & $60-64$ & & \\
\hline \multirow{2}{*}{$\begin{array}{c}\text { Male relatives } \\
3544 \\
45-54 \\
55-64 \\
65-74 \\
75 \text { and o:er } \\
\text { Female relatives } \\
35-44 \\
45-54 \\
55-64 \\
65-74 \\
75 \text { and over }\end{array}$} & $\begin{array}{r}288 \\
238 \\
154 \\
64 \\
12 \\
\end{array}$ & $\begin{array}{r}464 \\
377 \\
219 \\
123 \\
25\end{array}$ & $\begin{array}{c}330 \\
312.5 \\
209 \\
78 \\
15.5\end{array}$ & $\begin{array}{l}440 \\
460 \cdot 5 \\
410 \\
230 \\
46\end{array}$ & $\begin{array}{l}160 \\
160 \\
128 \cdot 5 \\
81 \\
12\end{array}$ & $\begin{array}{l}1682 \\
1548 \\
1120.5 \\
576 \\
110.5\end{array}$ & $\begin{array}{l}888.5 \\
950 \\
827.5 \\
460 \\
87.5\end{array}$ \\
\hline & $\begin{array}{l}351 \\
273 \cdot 5 \\
167.5 \\
96 \\
21\end{array}$ & $\begin{array}{c}410 \\
446 \cdot 5 \\
322 \cdot 5 \\
184 \\
54\end{array}$ & $\begin{array}{l}479 \\
436.5 \\
319 \\
186 \\
67\end{array}$ & $\begin{array}{c}340 \\
395 \\
365 \\
207 \cdot 5 \\
67\end{array}$ & $\begin{array}{c}132.5 \\
175 \\
183.5 \\
158.5 \\
48\end{array}$ & $\begin{array}{l}1712.5 \\
1726.5 \\
1357.5 \\
832 \\
257\end{array}$ & $\begin{array}{l}1046 \cdot 5 \\
1150 \\
966 \\
652 \cdot 5 \\
183\end{array}$ \\
\hline \multirow{4}{*}{$\begin{array}{c}\text { Male relatives } \\
35-44 \\
45-54 \\
55-64 \\
65-74 \\
75 \text { and over } \\
\text { Female relatives } \\
35-44 \\
45-54 \\
55-64 \\
65-74 \\
75 \text { and over }\end{array}$} & \multicolumn{5}{|c|}{ Female Patients } & & \multirow{2}{*}{$\underset{\text { (all ages) }}{\text { Female Control }}$} \\
\hline & \multirow{2}{*}{\multicolumn{3}{|c|}{$\begin{array}{c}5-64 \\
\\
655.5 \\
640 \\
504 \\
211 \cdot 5 \\
23.5 \\
\end{array}$}} & 65 and over & & & \\
\hline & & & & $\begin{array}{l}7.5 \\
20 \\
20 \\
17.5 \\
0\end{array}$ & & $\begin{array}{r}1436 \\
1288 \\
873 \\
380 \\
58\end{array}$ & $\begin{array}{c}1043 \cdot 5 \\
1000 \\
797 \\
469 \\
121\end{array}$ \\
\hline & $\begin{array}{c}188.5 \\
172.5 \\
102 \cdot 5 \\
54 \\
7.5\end{array}$ & $\begin{array}{c}458 \cdot 5 \\
435 \\
346 \\
176 \cdot 5 \\
64\end{array}$ & $\begin{array}{c}572.5 \\
567 \cdot 5 \\
504 \\
326.5 \\
98\end{array}$ & $\begin{array}{l}60 \\
80 \\
70 \\
60 \\
19\end{array}$ & & $\begin{array}{l}1279.5 \\
1255 \\
1022.5 \\
617 \\
188.5\end{array}$ & $\begin{array}{c}1493.5 \\
1327.5 \\
1070 \\
591 \\
199\end{array}$ \\
\hline
\end{tabular}


TABLE VA

OBSERVED NUMBER (O) OF DEATHS CERTIFIED TO BE DUE TO 'ARTERIOSCLEROTIC HEART DISEASE INCLUDING CORONARY DISEASE' (CATEGORIES 420 AND 94) BY AGE IN RELATIVES OF MALE INDEX PATIENTS, COMPARED WITH THE NUMBER EXPECTED (E) FROM THE REGISTER GENERAL'S TABLES

\begin{tabular}{|c|c|c|c|c|c|c|c|c|c|c|c|c|c|c|c|}
\hline \multirow{3}{*}{$\begin{array}{l}\text { Age of Relative } \\
\text { at Death From } \\
\text { Ischaemic } \\
\text { Heart Disease }\end{array}$} & \multicolumn{9}{|c|}{$\begin{array}{l}\text { 'Younger' Patients' Ages at Onset of } \\
\text { Ischaemic Heart Disease }\end{array}$} & \multicolumn{6}{|c|}{$\begin{array}{l}\text { 'Older' Patients' Ages at Onset of } \\
\text { Ischaemic Heart Disease }\end{array}$} \\
\hline & \multicolumn{3}{|c|}{$35-44$} & \multicolumn{3}{|c|}{$45-49$} & \multicolumn{3}{|c|}{$50-54$} & \multicolumn{3}{|c|}{$55-59$} & \multicolumn{3}{|c|}{60 and over } \\
\hline & $\mathrm{O}$ & E & O $E$ & $\mathrm{O}$ & E & $\mathbf{O} \mathbf{E}$ & $\mathrm{O}$ & $\mathbf{E}$ & $\mathbf{O} / \mathbf{E}$ & $\mathrm{O}$ & E & $\mathbf{O} / \mathbf{E}$ & $\mathrm{O}$ & E & $\mathbf{O} / \mathbf{E}$ \\
\hline $\begin{array}{c}\text { Male relatives } \\
35-44 \\
45-54 \\
\end{array}$ & $\mathbf{2}$ & $\begin{array}{l}0.08 \\
0.32\end{array}$ & $\begin{array}{r}25 \cdot 00 \\
3 \cdot 13 \\
\end{array}$ & I & $\begin{array}{l}0.12 \\
0.52\end{array}$ & $\begin{array}{l}8 \cdot 33 \\
5 \cdot 77 \\
\end{array}$ & $\begin{array}{l}0 \\
\text { I }\end{array}$ & $\begin{array}{l}0.06 \\
0.45\end{array}$ & $\overline{2 \cdot 22}$ & $\begin{array}{l}0 \\
2\end{array}$ & $\begin{array}{l}0.09 \\
0.64\end{array}$ & - & $\begin{array}{l}0 \\
0\end{array}$ & $\begin{array}{l}0.03 \\
0.22\end{array}$ & 二 \\
\hline $\begin{array}{l}55-64 \\
65-74 \\
75 \text { and over }\end{array}$ & $\begin{array}{l}4 \\
3 \\
0\end{array}$ & $\begin{array}{l}0.59 \\
0.61 \\
0.26\end{array}$ & $\begin{array}{l}6 \cdot 78 \\
4 \cdot 92 \\
-\end{array}$ & $\begin{array}{l}2 \\
2 \\
1\end{array}$ & $\begin{array}{l}0.81 \\
1 \cdot 11 \\
0.74\end{array}$ & $\begin{array}{l}2 \cdot 47 \\
1 \cdot 80 \\
1 \cdot 35\end{array}$ & $\begin{array}{l}2 \\
1 \\
0\end{array}$ & $\begin{array}{l}0.89 \\
0.54 \\
0.28\end{array}$ & $\begin{array}{l}2 \cdot 25 \\
1 \cdot 85 \\
-\end{array}$ & $\begin{array}{l}1 \\
4 \\
3\end{array}$ & $\begin{array}{l}\mathbf{I} \cdot 30 \\
1 \cdot 55 \\
0.87\end{array}$ & $\begin{array}{l}0 \cdot 77 \\
2 \cdot 58 \\
3 \cdot 45\end{array}$ & $\begin{array}{l}0 \\
0 \\
0\end{array}$ & $\begin{array}{l}0.52 \\
0.59 \\
0.19\end{array}$ & $\overline{-}$ \\
\hline $\begin{array}{l}\text { Female relatives } \\
\qquad \begin{array}{c}35-44 \\
45-54 \\
55-64\end{array}\end{array}$ & $\begin{array}{l}\circ \\
\circ \\
\circ\end{array}$ & $\begin{array}{l}0.01 \\
0.06 \\
0.18\end{array}$ & $\bar{E}$ & $\begin{array}{l}0 \\
0 \\
2\end{array}$ & $\begin{array}{l}0.02 \\
0.09 \\
0.28\end{array}$ & $\overline{7 \cdot 14}$ & $\begin{array}{l}0 \\
\text { I } \\
\text { O }\end{array}$ & $\begin{array}{l}0.02 \\
0.10 \\
0.33\end{array}$ & $\frac{-}{10 \cdot 00}$ & $\begin{array}{l}0 \\
0 \\
\mathbf{I}\end{array}$ & $\begin{array}{l}0.01 \\
0.09 \\
0.30\end{array}$ & $\bar{z}$ & $\begin{array}{l}\circ \\
0 \\
0\end{array}$ & $\begin{array}{l}- \\
0.03 \\
0.23\end{array}$ & $\overline{-}$ \\
\hline $\begin{array}{l}65-74 \\
75 \text { and over }\end{array}$ & $\begin{array}{l}\text { I } \\
0\end{array}$ & $\begin{array}{l}0.43 \\
0.26\end{array}$ & 2.33 & $\begin{array}{l}\text { I } \\
0\end{array}$ & $\begin{array}{l}0.67 \\
0.75\end{array}$ & $1 \cdot 49$ & $\begin{array}{l}2 \\
0\end{array}$ & $\begin{array}{l}0.69 \\
0.73\end{array}$ & 2.90 & $\begin{array}{l}\mathbf{I} \\
3\end{array}$ & $\begin{array}{l}0.61 \\
0.75\end{array}$ & $\begin{array}{l}r \cdot 64 \\
4 \cdot 00\end{array}$ & $\begin{array}{l}\mathbf{I} \\
\mathbf{2}\end{array}$ & $\begin{array}{l}0.60 \\
0.51\end{array}$ & $\begin{array}{l}1 \cdot 67 \\
3 \cdot 92\end{array}$ \\
\hline
\end{tabular}

Tables in categories 420 and 94 (see Appendix I) in each age-group. The third column $(\mathrm{O} / \mathrm{E})$ gives the ratio of observed to expected numbers of deaths. The findings are summarized in Table VI.

Tables VA and VB show details of the results and demonstrate a progressively diminishing relative risk to relatives with increasing age of both patients and relatives. The numbers in the small groups are too small to be statistically significant. Table VI, however, with its broader grouping shows that there is a significantly increased risk of death from ischaemic heart disease to first degree relatives of patients. The observed increase is $6 \frac{1}{2}$-fold amongst the 'younger' male relatives of the 'younger' female

TABLE VB

OBSERVED NUMBER (O) OF DEATHS CERTIFIED TO BE DUE TO 'ARTERIOSCLEROTIC HEART DISEASE INCLUDING CORONARY DISEASE' (CATEGORIES 420 AND 94) BY AGE IN RELATIVES OF FEMALE INDEX PATIENTS, COMPARED WITH THE NUMBER EXPECTED (E) FROM THE REGISTRAR GENERAL'S TABLES

\begin{tabular}{|c|c|c|c|c|c|c|c|c|c|c|c|c|}
\hline \multirow{3}{*}{$\begin{array}{c}\text { Age of Relative } \\
\text { at Death From } \\
\text { Ischaemic Heart } \\
\text { Disease }\end{array}$} & \multicolumn{9}{|c|}{ 'Younger' Patients' Ages at Onset of Ischaemic Heart Disease } & \multicolumn{3}{|c|}{$\begin{array}{c}\text { 'Older' Patients' Ages } \\
\text { at Onset of Ischaemic } \\
\text { Heart Disease }\end{array}$} \\
\hline & \multicolumn{3}{|c|}{$35-44$} & \multicolumn{3}{|c|}{$45-54$} & \multicolumn{3}{|c|}{$55-64$} & \multicolumn{3}{|c|}{65 and over } \\
\hline & O & $\mathrm{E}$ & $\mathrm{O} / \mathrm{E}$ & $\mathrm{O}$ & $\mathrm{E}$ & $\mathrm{O} / \mathrm{E}$ & $\mathrm{O}$ & $\mathrm{E}$ & $\mathrm{O} / \mathrm{E}$ & $\mathrm{O}$ & $\mathbf{E}$ & $\mathrm{O} / \mathrm{E}$ \\
\hline $\begin{array}{r}\text { Male relatives } \\
35-44 \\
45-54\end{array}$ & $\begin{array}{l}\mathbf{I} \\
\mathbf{2}\end{array}$ & $\begin{array}{l}0.06 \\
0.17\end{array}$ & $\begin{array}{l}16 \cdot 67 \\
11 \cdot 77\end{array}$ & $\begin{array}{l}3 \\
4\end{array}$ & $\begin{array}{l}0.15 \\
0.73\end{array}$ & $\begin{array}{r}20 \cdot 00 \\
5.48\end{array}$ & $\begin{array}{l}0 \\
3\end{array}$ & $\begin{array}{l}0.111 \\
0.80\end{array}$ & $\overline{3 \cdot 75}$ & $\begin{array}{l}\circ \\
0\end{array}$ & $\begin{array}{l}0.00 \\
0.01\end{array}$ & - \\
\hline $\begin{array}{l}55-64 \\
65-74 \\
75 \text { and over }\end{array}$ & $\begin{array}{l}\mathbf{I} \\
\mathbf{I} \\
\mathbf{0}\end{array}$ & $\begin{array}{l}0.24 \\
0.29 \\
0.26\end{array}$ & $\begin{array}{l}4 \cdot 17 \\
3.45 \\
-\end{array}$ & $\begin{array}{l}\mathbf{2} \\
\mathbf{I} \\
\mathbf{2}\end{array}$ & $\begin{array}{l}1 \cdot 52 \\
0.87 \\
0.37\end{array}$ & $\begin{array}{l}I \cdot 32 \\
1 \cdot 15 \\
5 \cdot 41\end{array}$ & $\begin{array}{l}4 \\
6 \\
1\end{array}$ & $\begin{array}{l}2 \cdot 20 \\
1 \cdot 72 \\
0 \cdot 40\end{array}$ & $\begin{array}{l}1 \cdot 82 \\
3 \cdot 49 \\
2 \cdot 50\end{array}$ & $\begin{array}{l}0 \\
\mathbf{I} \\
\mathbf{0}\end{array}$ & $\begin{array}{l}0.06 \\
0.14 \\
0.00\end{array}$ & $\overline{7 \cdot 14}$ \\
\hline $\begin{array}{r}\text { Female relatives } \\
35-44 \\
45-54 \\
55-64 \\
\end{array}$ & $\begin{array}{l}\mathbf{0} \\
\mathbf{I} \\
\mathbf{0}\end{array}$ & $\begin{array}{l}0.01 \\
0.04 \\
0.13\end{array}$ & $25 \cdot 00$ & $\begin{array}{l}0 \\
0 \\
2\end{array}$ & $\begin{array}{l}0.02 \\
0.09 \\
0.36\end{array}$ & $\frac{-}{5 \cdot 56}$ & $\begin{array}{l}0 \\
0 \\
6\end{array}$ & $\begin{array}{l}0.02 \\
0.12 \\
0.51\end{array}$ & $\overline{\text { II } 76}$ & $\begin{array}{l}\circ \\
\circ \\
0\end{array}$ & $\begin{array}{l}0.00 \\
0.02 \\
0.05\end{array}$ & E \\
\hline $\begin{array}{l}65-74 \\
75 \text { and over }\end{array}$ & $\begin{array}{l}\circ \\
0\end{array}$ & $\begin{array}{l}0.32 \\
0.09\end{array}$ & - & $\begin{array}{l}\mathbf{I} \\
\mathbf{0}\end{array}$ & $\begin{array}{l}0.58 \\
0.51\end{array}$ & $\underline{1.56}$ & $\begin{array}{l}\mathbf{I} \\
\mathbf{2}\end{array}$ & $\begin{array}{l}0.98 \\
0.83\end{array}$ & $\begin{array}{l}1 \cdot 02 \\
2 \cdot 41\end{array}$ & o & $\begin{array}{l}0.20 \\
0.19\end{array}$ & $\overline{5 \cdot 26}$ \\
\hline
\end{tabular}


TABLE VI

SUMMARY OF RISKS OF DEATH FROM ISCHAEMIC HEART DISEASE (REGISTRAR GENERAL'S CATEGORIES 420 AND 94) IN RELATIVES OF PATIENTS

\begin{tabular}{|c|c|c|c|c|c|c|}
\hline \multirow{3}{*}{$\begin{array}{l}\text { Age of Relative } \\
\text { at Death From } \\
\text { Ischaemic Heart } \\
\text { Disease }\end{array}$} & \multicolumn{6}{|c|}{ Male Patients' Ages at Onset of Ischaemic Heart Disease } \\
\hline & \multicolumn{3}{|c|}{$35-54$} & \multicolumn{3}{|c|}{55 and over } \\
\hline & $\mathrm{O}$ & $\mathbf{E}$ & $\mathrm{O} / \mathbf{E}$ & $\mathrm{O}$ & $\mathbf{E}$ & $\mathbf{O} / \mathbf{E}$ \\
\hline $\begin{array}{l}\text { Male relatives } \\
\qquad 55-54 \\
55 \text { and over }\end{array}$ & $\begin{array}{r}8 \\
15\end{array}$ & $\begin{array}{l}1.55 \\
5.83\end{array}$ & $\begin{array}{l}5 \cdot 16^{\star \star} \\
2 \cdot 57^{\star}\end{array}$ & $\begin{array}{l}2 \\
8\end{array}$ & $\begin{array}{l}0.98 \\
5.02\end{array}$ & $\begin{array}{l}2.04 \\
1 \cdot 59\end{array}$ \\
\hline $\begin{array}{l}\text { Female relatives } \\
\qquad \begin{array}{l}35-64 \\
65 \text { and over }\end{array}\end{array}$ & $\begin{array}{l}3 \\
4\end{array}$ & $\begin{array}{l}1.09 \\
3.53\end{array}$ & $\begin{array}{l}2 \cdot 75 \\
1 \cdot 13\end{array}$ & $\begin{array}{l}\mathbf{I} \\
7\end{array}$ & $\begin{array}{l}0.66 \\
2.47\end{array}$ & $\begin{array}{l}1 \cdot 52 \\
2 \cdot 83\end{array}$ \\
\hline & \multicolumn{6}{|c|}{ Female Patients' Ages at Onset of Ischaemic Heart Disease } \\
\hline & \multicolumn{3}{|c|}{$35-64$} & \multicolumn{3}{|c|}{65 and over } \\
\hline & $\mathbf{O}$ & $\mathrm{E}$ & $\mathbf{O} / \mathbf{E}$ & $\mathrm{O}$ & $\mathbf{E}$ & $\mathbf{O} / \mathbf{E}$ \\
\hline $\begin{array}{l}\text { Male relatives } \\
\qquad 55-54 \text { and over }\end{array}$ & $\begin{array}{l}13 \\
18\end{array}$ & $\begin{array}{l}2 \cdot 02 \\
7 \cdot 87\end{array}$ & $\begin{array}{l}6 \cdot 44^{\star \star} \\
2 \cdot 29^{\star}\end{array}$ & $\begin{array}{l}0 \\
\text { I }\end{array}$ & $\begin{array}{l}0.01 \\
0.20\end{array}$ & 5.00 \\
\hline $\begin{array}{l}\text { Female relatives } \\
\qquad \begin{array}{l}35-64 \\
65 \text { and over }\end{array}\end{array}$ & $\begin{array}{l}9 \\
4\end{array}$ & $\begin{array}{l}I \cdot 30 \\
3 \cdot 31\end{array}$ & $\begin{array}{l}6 \cdot 92^{\star \star} \\
I \cdot 2 I\end{array}$ & $\begin{array}{l}0 \\
1\end{array}$ & $\begin{array}{l}0.05 \\
0.39\end{array}$ & $-\overline{2 \cdot 56}$ \\
\hline
\end{tabular}

From the Poisson distribution (Pearson and Hartley, 1954) $\mathrm{p}<0.01$ shown $\star p<0.001$ shown $k \star$

TABLE VII

COMPARISON OF RISKS OF DEATH FROM MYOCARDIAL DEGENERATION IN RELATIVES OF PATIENTS AND GENERAL POPULATION USING REGISTRAR GENERAL'S CATEGORIES 93, 42I, AND 422

\begin{tabular}{|c|c|c|c|c|c|c|}
\hline \multirow{3}{*}{$\begin{array}{c}\text { Age of Relative } \\
\text { at Death From } \\
\text { Ischaemic Heart } \\
\text { Disease }\end{array}$} & \multicolumn{6}{|c|}{ Male Patients' Ages at Onset of Ischaemic Heart Disease } \\
\hline & \multicolumn{3}{|c|}{$35-54$} & \multicolumn{3}{|c|}{55 and over } \\
\hline & $\mathbf{O}$ & $\mathrm{E}$ & $\mathrm{O} / \mathrm{E}$ & o & $\mathrm{E}$ & $\mathrm{O} / \mathrm{E}$ \\
\hline $\begin{array}{l}\text { Male relatives } \\
\qquad \begin{array}{l}35-54 \\
55 \text { and over }\end{array}\end{array}$ & $\begin{array}{l}\circ \\
3\end{array}$ & $\begin{array}{l}0.37 \\
3.88\end{array}$ & $\overline{0.77}$ & $\begin{array}{l}0 \\
4\end{array}$ & $\begin{array}{l}0.27 \\
5.27\end{array}$ & $\overline{0.76}$ \\
\hline $\begin{array}{l}\text { Female relatives } \\
\qquad \begin{array}{l}35-64 \\
65 \text { and over }\end{array}\end{array}$ & I & $\begin{array}{l}1 \cdot 36 \\
5 \cdot 42\end{array}$ & $\begin{array}{l}0 \cdot 74 \\
0.18\end{array}$ & $\begin{array}{l}2 \\
3\end{array}$ & $\begin{array}{l}0.91 \\
4.93\end{array}$ & $\begin{array}{l}2 \cdot 20 \\
0.61\end{array}$ \\
\hline & \multicolumn{6}{|c|}{ Female Patients' Ages at Onset of Ischaemic Heart Disease } \\
\hline & \multicolumn{3}{|c|}{$35-64$} & \multicolumn{3}{|c|}{65 and over } \\
\hline & O & E & $\mathbf{O} / \mathbf{E}$ & $\mathrm{O}$ & E & $\mathrm{O} / \mathrm{E}$ \\
\hline $\begin{array}{l}\text { Male relatives } \\
\\
\qquad 5-54 \\
55 \text { and over }\end{array}$ & $\begin{array}{l}2 \\
8\end{array}$ & $\begin{array}{l}0.63 \\
3.90\end{array}$ & $\begin{array}{l}3.17 \\
2.05\end{array}$ & $\begin{array}{l}0 \\
\text { I }\end{array}$ & $\begin{array}{l}0.01 \\
0.43\end{array}$ & $-\overline{2 \cdot 33}$ \\
\hline $\begin{array}{l}\text { Female relatives } \\
\qquad \begin{array}{l}35-64 \\
65 \text { and over }\end{array}\end{array}$ & $\begin{array}{r}0 \\
12\end{array}$ & $\begin{array}{l}1 \cdot 48 \\
7 \cdot 77\end{array}$ & $\overline{\mathrm{r} \cdot 54}$ & $\circ$ & $\begin{array}{l}0.13 \\
0.85\end{array}$ & $\bar{Z}$ \\
\hline
\end{tabular}


TABLE VIII

COMPARISON OF RISKS OF DEATH FROM CEREBRAL HAEMORRHAGE AND THROMBOSIS IN RELATIVES OF PATIENTS AND GENERAL POPULATION USING REGISTRAR GENERAL'S CATEGORIES 97'1, 82, 83, 331 , AND 332

\begin{tabular}{|c|c|c|c|c|c|c|}
\hline \multirow{3}{*}{$\begin{array}{l}\text { Age of Relative } \\
\text { at Death From } \\
\text { Cerebral Haemorrhage } \\
\text { and Thrombosis }\end{array}$} & \multicolumn{6}{|c|}{ Male Patients' Ages at Onset of Ischaemic Heart Disease } \\
\hline & \multicolumn{3}{|c|}{$35-54$} & \multicolumn{3}{|c|}{55 and over } \\
\hline & $\mathbf{O}$ & $\mathbf{E}$ & $\mathbf{O} / \mathbf{E}$ & $\mathbf{O}$ & $\mathbf{E}$ & $\mathbf{O} / \mathbf{E}$ \\
\hline $\begin{array}{l}\text { Male relatives } \\
\qquad 55-54 \\
55 \text { and over }\end{array}$ & $\begin{array}{l}2 \\
2\end{array}$ & $\begin{array}{l}0.53 \\
3.81\end{array}$ & $\begin{array}{l}3.77 \\
0.52\end{array}$ & $\begin{array}{l}0 \\
3\end{array}$ & $\begin{array}{l}0.35 \\
4.27\end{array}$ & $\overline{0.70}$ \\
\hline $\begin{array}{l}\text { Female relatives } \\
\qquad \begin{array}{l}35-64 \\
65 \text { and over }\end{array}\end{array}$ & $\begin{array}{l}6 \\
5\end{array}$ & $\begin{array}{l}2 \cdot 31 \\
5 \cdot 71\end{array}$ & $\begin{array}{l}2.60 \\
0.88\end{array}$ & $\begin{array}{l}I \\
4\end{array}$ & $\begin{array}{l}I \cdot 42 \\
4 \cdot 44\end{array}$ & $\begin{array}{l}0.70 \\
0.90\end{array}$ \\
\hline & \multicolumn{6}{|c|}{ Female Patients' Ages at Onset of Ischaemic Heart Disease } \\
\hline & \multicolumn{3}{|c|}{$35-64$} & \multicolumn{3}{|c|}{65 and over } \\
\hline & $\mathbf{O}$ & $\mathbf{E}$ & $\mathbf{O} / \mathbf{E}$ & $\mathbf{O}$ & $\mathbf{E}$ & $\mathrm{O} / \mathrm{E}$ \\
\hline $\begin{array}{l}\text { Male relatives } \\
\qquad \begin{array}{l}35-54 \\
55 \text { and over }\end{array}\end{array}$ & $\begin{array}{l}3 \\
8\end{array}$ & $\begin{array}{l}0.73 \\
4.87\end{array}$ & $\begin{array}{l}4 \cdot I I \\
I \cdot 64\end{array}$ & $\begin{array}{l}0 \\
\text { I }\end{array}$ & $\begin{array}{l}0.01 \\
0.09\end{array}$ & $\overline{I I \cdot I I}$ \\
\hline $\begin{array}{l}\text { Female relatizes } \\
\qquad \begin{array}{l}35-64 \\
65 \text { and over }\end{array}\end{array}$ & $\begin{array}{r}3 \\
12\end{array}$ & $\begin{array}{l}2 \cdot 29 \\
6 \cdot 11\end{array}$ & $\begin{array}{l}I \cdot 3 I \\
I \cdot 96\end{array}$ & $\begin{array}{l}0 \\
2\end{array}$ & $\begin{array}{l}0.16 \\
0.73\end{array}$ & $\overline{2 \cdot 74}$ \\
\hline
\end{tabular}

patients, 7-fold amongst their 'younger' female relatives, and 5-fold amongst the 'younger' male relatives of the 'younger' male patients. These increases are all significant at the $I$ in rooo level (Pearson and Hartley, 1954). Amongst the 'older' male relatives of all the 'younger' patients the risks are doubled $(p=<0.01)$, while among the 'younger' female relatives of the 'younger' male patients the risk is doubled, though the figure is not significant.

Risks of Death from Myocardial Degeneration, Cerebrovascular Disorder and All Causes in Patients' Relatives. Table VII shows a summary of deaths observed $(\mathrm{O})$ and expected $(\mathrm{E})$ and the ratio $(\mathrm{O} / \mathrm{E})$ from myocardial degeneration, Registrar General's categories 93 in I $931-49$ and 421 and 422 from 1950, and shows no indication of any increase in the number of deaths certified in categories 421,422 , and 93 in the female relatives of patients with ischaemic heart disease.

Table VIII shows a similar summary of cerebrovascular disorder in patients' relatives, using the Registrar General's categories 82a and b, and 97. I in 1931-39, 83a, b, and c in 1940-49, and 331 and 332 from 1950. The expected numbers and observed numbers are too small for significant com- parison when considered in four groups divided by sex, but when comparison is made between the sum of the expected $(5.86)$ and observed numbers (14) in the 'younger' relatives of the 'younger' patients there is a more than 2-fold increase which is significant $(p=<0.01)$.

Table IX shows a summary of the risks of death from all causes minus deaths from coronary artery disease, Registrar General's categories 94 in 1931-49 and 420 in 1950-present. When the risks of dying from coronary artery disease are subtracted from the risks of dying from all causes, the observed numbers of deaths are not significantly different from the expected numbers.

Risks of Death from Ischaemic Heart Disease and Cerebrovascular Disorders in Relatives of Controls. Table $\mathrm{X}$ shows the observed and expected deaths from ischaemic heart disease (Registrar General's categories 420 and 94) and from cerebral harmorrhage and thrombosis (Registrar General's categories $97 \cdot \mathrm{r}, 82 \mathrm{a}$ and b, 83a, b, and c, 33I and 332) in the relatives of controls. The deaths observed from both ischaemic heart disease and cerebrovascular disorder in the relatives of the control series are close to those expected from the incidence in the general population. It is, therefore, 
TABLE IX

COMPARISON OF RISKS OF DEATH FROM ALL CAUSES (EXCLUDING DEATHS IN REGISTRAR GENERAL'S CATEGORIES 420 AND 94) IN RELATIVES OF PATIENTS AND GENERAL POPULATION

\begin{tabular}{|c|c|c|c|c|c|c|}
\hline \multirow{3}{*}{ Age of Relative at Death } & \multicolumn{6}{|c|}{ Male Patients' Ages at Onset of Ischaemic Heart Disease } \\
\hline & \multicolumn{3}{|c|}{$35-54$} & \multicolumn{3}{|c|}{55 and over } \\
\hline & $\mathrm{O}$ & $\mathrm{E}$ & $\mathbf{O} / \mathbf{E}$ & $\mathrm{O}$ & $\mathrm{E}$ & $\mathrm{O} / \mathrm{E}$ \\
\hline $\begin{array}{l}\text { Male relatives } \\
\qquad 55-54 \\
55 \text { and over }\end{array}$ & $\begin{array}{r}9 \\
27\end{array}$ & $\begin{array}{l}10 \cdot 111 \\
29 \cdot 02\end{array}$ & $\begin{array}{l}0.89 \\
0.93\end{array}$ & $\begin{array}{r}2 \\
31\end{array}$ & $\begin{array}{r}6 \cdot 51 \\
32 \cdot 37\end{array}$ & $\begin{array}{l}0.3 I \\
0.96\end{array}$ \\
\hline $\begin{array}{l}\text { Female relatives } \\
\qquad \begin{array}{l}35-64 \\
65 \text { and over }\end{array}\end{array}$ & $\begin{array}{l}\text { I } 4 \\
3 \text { I }\end{array}$ & $\begin{array}{l}19 \cdot 44 \\
27 \cdot 48\end{array}$ & $\begin{array}{l}0.72 \\
1.13\end{array}$ & $\begin{array}{l}10 \\
26\end{array}$ & $\begin{array}{l}I I \cdot 72 \\
22 \cdot 58\end{array}$ & $\begin{array}{l}0.85 \\
I .15\end{array}$ \\
\hline & \multicolumn{6}{|c|}{ Female Patients' Ages at Onset of Ischaemic Heart Disease } \\
\hline & \multicolumn{3}{|c|}{$35-64$} & \multicolumn{3}{|c|}{65 and over } \\
\hline & $\mathrm{O}$ & $\mathbf{E}$ & $\mathrm{O} / \mathrm{E}$ & $\mathrm{O}$ & $\mathbf{E}$ & $\mathrm{O} / \mathrm{E}$ \\
\hline $\begin{array}{l}\text { Male relatives } \\
\qquad 55-54 \\
55 \text { and over }\end{array}$ & $\begin{array}{l}15 \\
39\end{array}$ & $\begin{array}{l}14 \cdot 17 \\
39 \cdot 67\end{array}$ & $\begin{array}{l}1.06 \\
0.93\end{array}$ & $\begin{array}{l}0 \\
3\end{array}$ & $\begin{array}{l}0.23 \\
0.83\end{array}$ & $\overline{3 \cdot 61}$ \\
\hline $\begin{array}{l}\text { Female relatives } \\
\qquad \begin{array}{l}35-64 \\
65 \text { and over }\end{array}\end{array}$ & $\begin{array}{l}20 \\
46\end{array}$ & $\begin{array}{l}21 \cdot 51 \\
34 \cdot 01\end{array}$ & $\begin{array}{l}0.93 \\
1.35\end{array}$ & $\begin{array}{l}0 \\
5\end{array}$ & $\begin{array}{l}I \cdot 56 \\
3 \cdot 72\end{array}$ & $\overline{1 \cdot 34}$ \\
\hline
\end{tabular}

TABLE X

COMPARISON OF RISKS OF DEATH IN RELATIVES OF CONTROLS FROM:

ISCHAEMIC HEART DISEASE (R.G.'S CATEGORIES 420 AND 94) AND FROM CEREBRAL HAEMORRHAGE AND THROMBOSIS (R.G.'S CATEGORIES 97.I, 82, 83, 33I, AND 332

\begin{tabular}{|c|c|c|c|c|c|c|}
\hline \multirow[t]{2}{*}{ Age at Death } & \multicolumn{3}{|c|}{$\begin{array}{l}\text { Deaths of Relatives } \\
\text { From Ischaemic Heart Disease }\end{array}$} & \multicolumn{3}{|c|}{$\begin{array}{l}\text { Deaths of Relatives } \\
\text { From Cerebral Haemorrhage }\end{array}$} \\
\hline & o & $\mathrm{E}$ & $\mathrm{O} / \mathrm{E}$ & $\mathrm{O}$ & $\mathrm{E}$ & $\mathrm{O} / \mathrm{E}$ \\
\hline $\begin{array}{ll}\text { Male relatives } & \\
& 35-54 \\
& 55 \text { and over }\end{array}$ & $\begin{array}{r}4 \\
12\end{array}$ & $\begin{array}{l}\text { I.20 } \\
9 \cdot 15\end{array}$ & $\begin{array}{l}3.33 \\
1 \cdot 31\end{array}$ & $\begin{array}{l}0 \\
5\end{array}$ & $\begin{array}{l}0.49 \\
6.61\end{array}$ & $\overline{0.76}$ \\
\hline $\begin{array}{l}\text { Female relatives } \\
\qquad \begin{array}{l}35-64 \\
65 \text { and over }\end{array}\end{array}$ & $\begin{array}{l}\mathrm{I} \\
6\end{array}$ & $\begin{array}{l}1.17 \\
5.40\end{array}$ & $\begin{array}{l}0.85 \\
1 \cdot 11\end{array}$ & $\begin{array}{l}\text { I } \\
5\end{array}$ & $\begin{array}{l}2.39 \\
7.78\end{array}$ & $\begin{array}{l}0.42 \\
0.64\end{array}$ \\
\hline & \multicolumn{6}{|c|}{ Female Controls (all ages) } \\
\hline & $\mathrm{o}$ & $\mathrm{E}$ & $\mathrm{O} / \mathrm{E}$ & $\mathrm{o}$ & E & $\mathrm{O} / \mathrm{E}$ \\
\hline $\begin{array}{l}\text { Male relatives } \\
\\
\text { 55 } 55 \text { and over }\end{array}$ & $\begin{array}{l}\text { O } \\
\text { II }\end{array}$ & $\begin{array}{l}1.47 \\
6.36\end{array}$ & $\overline{1 \cdot 73}$ & $\begin{array}{l}\circ \\
5\end{array}$ & $\begin{array}{l}0.56 \\
7.00\end{array}$ & $\overrightarrow{0.71}$ \\
\hline $\begin{array}{l}\text { Female relatives } \\
\qquad \begin{array}{l}35-64 \\
65 \text { and over }\end{array}\end{array}$ & $\begin{array}{l}1 \\
7\end{array}$ & $\begin{array}{l}1.31 \\
5.04\end{array}$ & $\begin{array}{l}0.76 \\
1.39\end{array}$ & $\begin{array}{l}2 \\
5\end{array}$ & $\begin{array}{l}2.46 \\
7.70\end{array}$ & $\begin{array}{l}0.81 \\
0.65\end{array}$ \\
\hline
\end{tabular}


not unreasonable to compare morbidity in the relatives of the patients with the controls.

Morbidity amongst Living Relatives of Patients and Controls. Table XI shows the incidence of ischaemic heart disease and confirmed diabetes amongst living relatives (all ages included) of patients and controls. The pattern of increased incidence of ischaemic heart disease amongst the living relatives of patients follows closely the pattern of increased risk of death. A higher incidence of diabetes is found amongst the relatives of female patients compared with controls.

TABLE XI

INCIDENCE OF ISCHAEMIC HEART DISEASE AND DIABETES AMONGST LIVING RELATIVES OF PATIENTS AND CONTROLS

\begin{tabular}{|c|c|c|c|}
\hline & Total & $\begin{array}{c}\text { Ischaemic } \\
\text { Heart } \\
\text { Disease }\end{array}$ & Diabetes \\
\hline $\begin{array}{l}\text { Male relatives of: } \\
\text { Male controls } \\
\text { Male patients }\end{array}$ & $\begin{array}{r}98 \\
162\end{array}$ & $\begin{array}{l}1 \\
8\end{array}$ & $\begin{array}{l}\text { I } \\
0\end{array}$ \\
\hline $\begin{array}{l}\text { Female relatives of: } \\
\text { Male controls } \\
\text { Male patients }\end{array}$ & $\begin{array}{l}139 \\
184\end{array}$ & $\begin{array}{l}0 \\
\text { I }\end{array}$ & $\begin{array}{l}1 \\
4\end{array}$ \\
\hline $\begin{array}{l}\text { Male relatives of } \\
\text { Female controls } \\
\text { Female patients }\end{array}$ & $\begin{array}{l}\text { I IO } \\
\text { I } 21\end{array}$ & $\begin{array}{r}2 \\
11\end{array}$ & $\begin{array}{l}1 \\
6\end{array}$ \\
\hline $\begin{array}{l}\text { Female relatives of: } \\
\text { Female controls } \\
\text { Female patients }\end{array}$ & $\begin{array}{l}172 \\
\text { I } 10\end{array}$ & $\begin{array}{l}0 \\
7\end{array}$ & $\begin{array}{l}0 \\
4\end{array}$ \\
\hline
\end{tabular}

Consanguinity in Index Patients and Controls. The parents of 2 unrelated female index patients were first cousins (see Appendix IIB, families 5I and 94). Both index patients had hypercholesterolaemic xanthomatosis. No consanguinity was found amongst the parents of the controls.

Twins. One male index patient has an identical twin who developed ischaemic heart disease 18 months after the onset in his brother. One male index patient has a twin sister who is well (see Appendix IIA, families IO2 and III).

\section{Discussion}

Ischaemic heart disease is a common cause of death, especially among men, and the risks have been increasing for men and women over the past 30 years. Male relatives in our series living through the ages 35-54 encountered an average expected risk of death from ischaemic heart disease of $I$ in 65 (calculated from the Registrar General's tables categories 420 and 94), and female relatives living through the ages 35-64 encountered a risk of $I$ in 90 .

The risks observed in our 'younger' series (to the nearest whole number) for male relatives of male index patients are $I$ in 12 , for male relatives of female index patients $I$ in 10 , for female relatives of male patients $I$ in 36 and for female relatives of female patients $I$ in 12 . This represents an approxi-

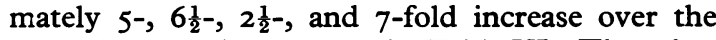
general population, as seen in Table VI. There has been a substantial increase in risk in the general population during the period under review, but the series is not large enough to see if the relative increase in risk remains constant. There is, however, a suggestion that as the general risk increases the relative risk drops. Taking all relatives together, between 1930 and 1945 there were 7 deaths when 0.6 would have been expected, whereas from 1945 to 1964,27 deaths occurred when 4.9 would have been expected.

The female relatives of male patients show substantially less increase in risk of death from ischaemic heart disease than the other three classes of relatives. There is no indication that this is due to a reluctance to diagnose death from 'coronary artery disease' in young women, since there is no surplus of deaths of female relatives in categories 42I, 422, and 93. This suggests that the familial causes (whether genetic or common family environment) of ischaemic heart disease in many men are not such as to cause ischaemic heart disease in women; the causes in women, however, can certainly affect their brothers and fathers.

The increased deaths from cerebral haemorrhage and thrombosis in the sum of the 4 classes of 'younger' relatives show that some aetiological factors are common in this group of disorders and in ischaemic heart disease. We were not, however, able to confirm Rose's observation (1964) that relatives of patients with ischaemic heart disease tend to die earlier from 'all causes', but, unlike Rose's, our observations are restricted to adult life.

Familial concentrations may be due to genetic resemblance between relatives, to common family environment or, as is probably the case here, a mixture of both. Members of a family will tend to share environmental factors such as occupational class, diet, smoking, and exercise habits which have been shown to be related to the incidence of ischaemic heart disease. This will perhaps be true of sibs rather more than of parent and child. It is not easy to compare risks between parents and sibs of patients in this series, because deaths occurring a generation apart are subject to differences in both risks and classification. It seems likely, however, 
COMPARISON OF RISKS OF DEATH IN R.G.'S CATEGORIES 420 AND 94 OF RELATIVES OF 'YOUNGER' FEMALE PATIENTS OF TOTAL GROUP (A) WITH THIS GROUP LESS RELATIVES OF 7 PATIENTS WITH HYPERCHOLESTEROLAEMIC XANTHOMATOSIS (B)

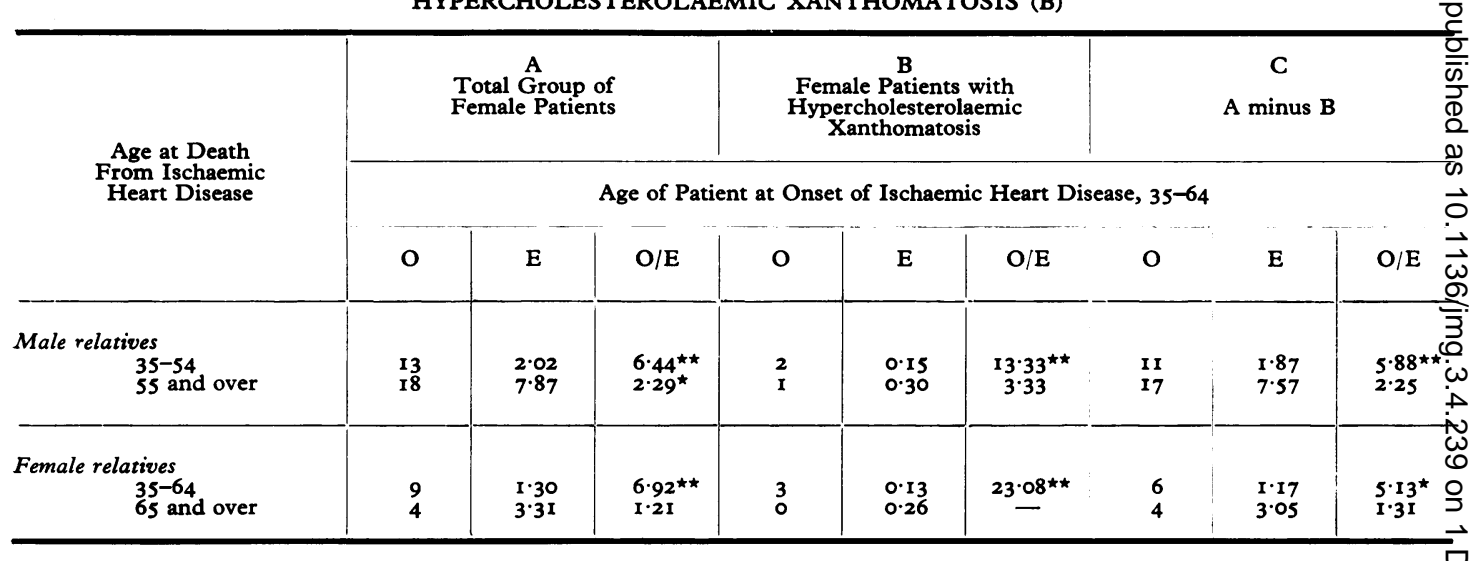

From the Poisson distribution (Pearson and Hartley, 1954) $p<0.01$ shown $\star p<0.001$ shown $\star \star$.

that the causes of the differences in risk to the female relatives of the male patients compared with female relatives of female patients are more likely to be genetic than environmental. A real distinction between common genes and common environment is perhaps best established by twin studies, and it would be of much interest to see the further results of the Danish twin study (Harvald and Hauge, I963), particularly for deaths from ischaemic heart disease in the 'younger' age-group. Meanwhile, if Falconer's method of analysis of heritability (1965) is applied to this survey the risks to men would be compatible with a heritability of $60 \%$ and for women of $70 \%$; though, as Falconer notes, the true heritability would be lower than this to the extent that the family concentrations are due to the common environment.

The index patients in this series were an unselected group with ischaemic heart disease and the familial concentrations might well vary according to the underlying causes. Exceptional familial concentrations of ischaemic heart disease are noted more frequently amongst families of the female than of the male patients (see Appendix II). There were only two striking familial concentrations among the families of the male patients (see Appendix IIA, families 40 and 120). In both, the mother was affected, and in family 40 the index patient has hypertriglyceridaemia. Familial concentrations appear more frequently amongst the female patients (see Appendix IIB, families I, 5I, 83, 84, and 93). In families $I, 5 I$, and 84 the index patients and one or more relatives have hypercholesterolaemic xanthomatosis; in family 84 , twin brothers of the index patient are reported, one died at 38 of ischaemio heart disease and the other at the same age wa9 found to have ischaemic heart disease and hyper $\vec{\theta}$ cholesterolaemic xanthomatosis; family 83 has family members with diabetes mellitus while in family 93 no special factors were found.

Index patients with hypercholesterolameic xans thomatosis form one specific subgroup worth specia study. In this series hypercholesterolaemic xantho@ matosis was diagnosed in 7 women patients; how ever, no systematic search was made for this con $\overrightarrow{\bar{\circ}}$ dition so it may have been present in more. Table of risk constructed for the family members of the $\bar{Z}$ female index patients diagnosed as having hypero cholesterolaemic xanthomatosis (see Table XI showed a 13-fold increase in risk to their youngô male relatives and a 23 -fold increase in risk to the 'younger' female relatives, indicating a specially high risk to the 'younger' relatives in these families When the life experience of the relatives of these hypercholesterolaemic families is subtracted frong the total series very little reduction in risk is show amongst the male relatives over the general popu을 lation; the female relatives show rather mork reduction in the increased risk over the unselected series.

\section{Summary}

The health experience of all adult first degres relatives of 121 men and 96 women with ischaemi heart disease and 104 men and 105 women controls? is reported. The causes of death and morbidity off both groups of relatives have been documented, an $\bar{\phi}$ causes of death (from death certificates) have beep 
classified by the Registrar General's staff using the criteria current at the date of death. A comparison has been made of deaths from ischaemic heart disease in the relatives of controls and the general population for England and Wales with the relatives of patients with ischaemic heart disease. When deaths under 55 years in men and 65 years in women are considered the relatives of both sexes of female patients with onset under 65 years show a nearly 7-fold increase compared with the general population and the male relatives of the male patients with onset under 55 years show an increase of risk of death which is 5 times that in the general population. The female relatives of the male patients experience an increased risk which is $2 \frac{1}{2}$ times that of the women in the general population.

Family concentrations of ischaemic heart disease are noted especially in the families of the female patients and are most marked in the families of patients with hypercholesterolaemic xanthomatosis or amongst families containing members with diabetes. Two pairs of twins with ischaemic heart disease are mentioned.

The evidence points to an increased risk of ischaemic heart disease to relatives and suggests that the increased risk may be in part due to genetic factors.

We thank Dr C. O. Carter for guidance throughout the study; Dr J. A. Fraser Roberts, F.R.S., for encouragement in the initial stages; the Physicians of the Central Middlesex, the Elizabeth Garrett Anderson, the Middlesex, and the National Heart Hospitals who allowed us to question their patients, and the many practitioners and records officers who replied to our letters. We are much indebted to the Registrar General for classifying the death certificates. We are indebted to the patients and controls who allowed us to trace their relatives and especially the Prudential Assurance Company for their collaboration.

\section{REFERENCES}

Falconer, D. S. (1965). The inheritance of liability to certain diseases, estimated from the incidence among relatives. Ann. hum. Genet., 29, 5 I.

Gertler, M. M., and White, P. D. (1954). Coronary Heart Disease in Young Adults; a Multidisciplinary Study. Harvard University Press, Cambridge, Massachusetts.

Harvald, B., and Hauge, M. (1963). Hereditary factors elucidated by twin studies. In Genetics and the Epidemiology of Chronic Disease, pp. 61-76. (U.S. Public Health Service Publication No. 1 I03), Washington, D.C.

Pearson, E. S., and Hartley, H. O. (1954). Biometrika Tables for Statisticians, Vol. I, p. I22. Cambridge University Press, London. Registrar General (1908-66). Statistical Review of England and Wales. Part I, Tables, medical. H.M.S.O., London.

Rose, G. (1964). Familial patterns in ischaemic heart disease. Brit. F. prev. soc. Med., 18, 75 .

Thomas, C. B., and Cohen, B. H. (1955). The familial occurrence of hypertension and coronary artery disease, with observations concerning obesity and diabetes. Ann. intern. Med., 42, 90.

\section{Appendix I}

Definitions of Categories used on Life Tables
from Registrar General's Statistical Review

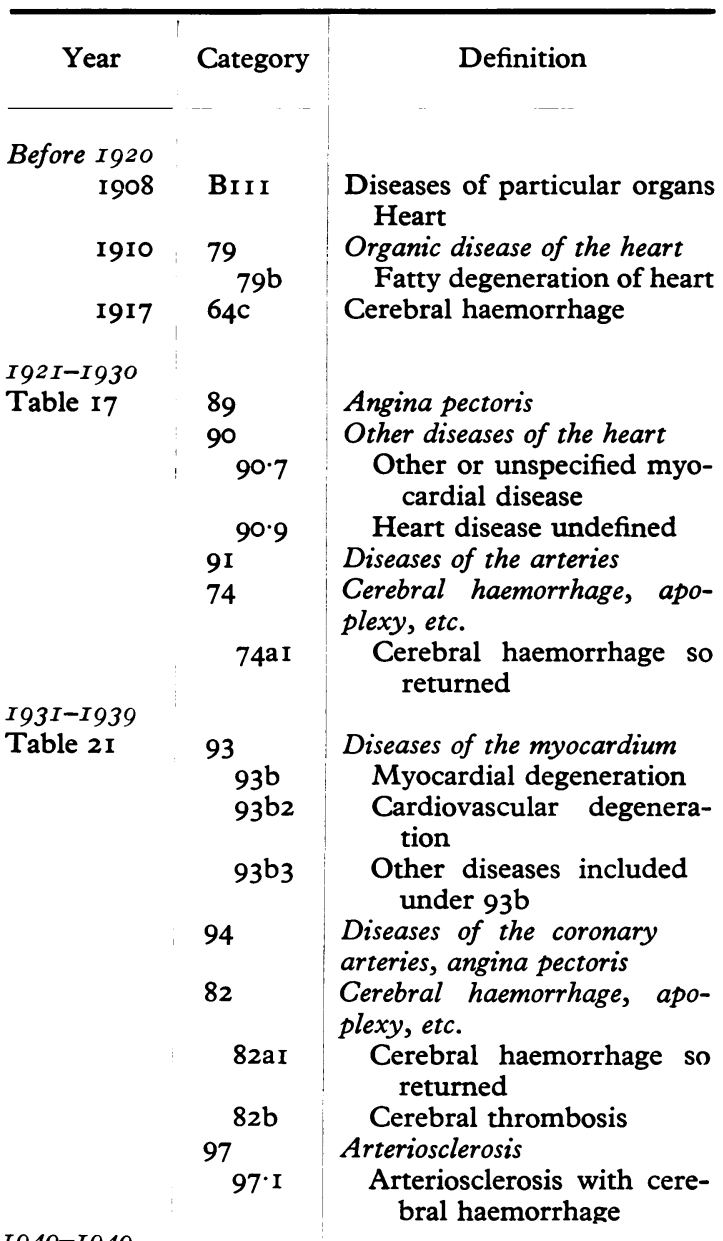

$-1949$

Table 2 I

93

Diseases of the myocardium

Myocardial degeneration, infarction, and sclerosis and other chronic myocarditis

Cardiovascular degeneration

Other myocardial degeneration

Myocarditis, not distinguished as acute or chronic

Diseases of the coronary

arteries, angina pectoris

$94 a$ 
Appendix I-continued

\begin{tabular}{|c|c|c|}
\hline Year & Category & Definition \\
\hline $\begin{array}{l}\text { I950-present } \\
\text { day } \\
\text { Table I7 }\end{array}$ & $\begin{array}{l}420-422 \\
420 \\
420 \cdot 1 \\
421 \\
422 \\
\quad 422 \cdot 1 \\
\quad 422 \cdot 2 \\
331 \\
332\end{array}$ & $\begin{array}{l}\text { Arteriosclerotic and degenera- } \\
\text { tive heart disease } \\
\text { Arteriosclerotic heart disease, } \\
\text { including coronary disease } \\
\text { Heart disease specified as } \\
\text { involving coronary } \\
\text { arteries } \\
\text { Chronic endocarditis not speci- } \\
\text { fied as rheumatic } \\
\text { Other myocardial degeneration } \\
\text { Myocardial degeneration } \\
\text { with arteriosclerosis } \\
\text { Other diseases included } \\
\text { under } 422 \\
\text { Cerebral haemorrhage } \\
\text { Cerebral embolism and throm- } \\
\text { bosis }\end{array}$ \\
\hline
\end{tabular}

\section{Appendix II}

\section{Details of families of index patients with ischaemic heart disease}

Male and female Ist degree relatives are shown M F; male and female half-sibs $\mathrm{m} f$; italics show relative affectef: with ischaemic heart disease, when followed by SP indicates affected relative diagnosed since pedigree was take and not used in tables; $\star_{\text {indicates diabetes; } † \text { hypercholesterolaemic xanthoma; CVD cerebrovascular disorder } \bar{S}}$ ND death not documented; RG's category following date of death shows classification by RG's staff (see Appendix I) $\mathrm{Mx}$ indicates male died at $\mathrm{x}$ years; round brackets indicate sibs under 20 years and half-sibs who do not appear o 8 tables; square brackets indicate twins; IE indicates insufficient evidence for use in tables; W indicates war death.

\begin{tabular}{|c|c|c|c|c|c|c|}
\hline \multirow[b]{2}{*}{$\begin{array}{l}\text { Family } \\
\text { No. }\end{array}$} & \multicolumn{2}{|c|}{ Propositi } & \multicolumn{2}{|c|}{ Parents } & \multirow[b]{2}{*}{ Sibs and half-sibs } & \multirow[b]{2}{*}{ Children } \\
\hline & $\begin{array}{l}\text { Year } \\
\text { of } \\
\text { Birth }\end{array}$ & $\begin{array}{c}\text { Age } \\
\text { at } \\
\text { Onset } \\
\text { IHD } \\
\end{array}$ & Mothers & Fathers & & \\
\hline \multicolumn{3}{|c|}{ A-MALE PATIENTS } & & & & \\
\hline \multicolumn{2}{|c|}{ One-child families } & & & & & \\
\hline $\begin{array}{l}\mathbf{I} \\
\mathbf{2} \\
\mathbf{3}\end{array}$ & $\begin{array}{l}1907 \\
1930 \\
1911\end{array}$ & $\begin{array}{l}46 \\
34 \\
50\end{array}$ & $\begin{array}{l}188 \mathrm{r}-1918 \\
1905 \\
1887\end{array}$ & $\begin{array}{l}1879-1962 \mathrm{ND} \\
1903 \\
1886-1955 \\
420.1\end{array}$ & & $\begin{array}{l}\text { Mr950 } \\
\text { Mr957; Mr959 } \\
\text { FI940; MI945; Mr948 }\end{array}$ \\
\hline $\begin{array}{l}4 \\
5\end{array}$ & $\begin{array}{l}1906 \\
1914\end{array}$ & $\begin{array}{l}57 \\
47\end{array}$ & $\begin{array}{l}1878-1935 \\
1878-1960\end{array}$ & $\begin{array}{l}1874-1910 \\
1869-1947 \\
94 a\end{array}$ & & $\begin{array}{l}\text { MI933 } \\
\text { MI942; Mr945; Fr949 }\end{array}$ \\
\hline 6 & 1912 & 48 & $\underset{83 a}{1881-1943}$ & $\begin{array}{c}188 I-19.53 \\
422.2\end{array}$ & & \\
\hline
\end{tabular}


Appendix I-continued

\begin{tabular}{|c|c|c|c|c|c|c|}
\hline \multirow[b]{2}{*}{$\begin{array}{l}\text { Family } \\
\text { No. }\end{array}$} & \multicolumn{2}{|c|}{ Propositi } & \multicolumn{2}{|c|}{ Parents } & \multirow[b]{2}{*}{ Sibs and half-sibs } & \multirow[b]{2}{*}{ Children } \\
\hline & $\begin{array}{l}\text { Year } \\
\text { of } \\
\text { Birth }\end{array}$ & $\begin{array}{l}\text { Age } \\
\text { at } \\
\text { Onset } \\
\text { IHD }\end{array}$ & Mothers & Fathers & & \\
\hline \multicolumn{7}{|c|}{ One-child families (continued) } \\
\hline 7 & 1913 & 50 & $\begin{array}{c}1890-1956 \\
420.1\end{array}$ & 1887 & & Mr940; Fr942; Mr946 \\
\hline 8 & I9II & 49 & $\begin{array}{c}1878-1948 \\
83 a\end{array}$ & $1872-1916$ & & Mr947; Mrg52; (Mo; Fo) \\
\hline $\begin{array}{r}9 \\
\text { IO }\end{array}$ & $\begin{array}{c}1912 \\
1906-1965\end{array}$ & $\begin{array}{l}48 \\
54\end{array}$ & $\begin{array}{l}1871-1947 \\
1967-1940 \mathrm{ND}\end{array}$ & $\begin{array}{l}1877-1915 W \\
1872-1931\end{array}$ & $\begin{array}{l}\text { (Mat. } \frac{1}{2} \text { sibs } f, f, f, f, m, m, m, \\
\text { 899-1944) }\end{array}$ & $\begin{array}{l}\text { Fr936 } \\
\text { Fr927; Fr937 }\end{array}$ \\
\hline I I & 1904 & 56 & $1870-1937$ & I 868-I944 & & $\begin{array}{l}\text { Fig36; Mrg40; Mr943; } \\
\text { (Mo; Mo; Fo) }\end{array}$ \\
\hline 12 & 1906 & 57 & $\begin{array}{l}\text { I878-1955 } \\
331\end{array}$ & $\begin{array}{c}1877-1942 \\
83 a\end{array}$ & & Mi935 \\
\hline \multicolumn{7}{|c|}{ Two-child families } \\
\hline $\begin{array}{l}13 \\
14\end{array}$ & $\begin{array}{l}1913 \\
\text { I9II }\end{array}$ & $\begin{array}{l}49 \\
44\end{array}$ & $\begin{array}{l}\text { I882-I } 960 N D \\
\text { I } 889\end{array}$ & $\begin{array}{l}1881-1941 \\
1878-1944 \\
94 a\end{array}$ & $\begin{array}{l}\text { Fig15 } \\
\text { Mig20 }\end{array}$ & $\begin{array}{l}\text { FI94I } \\
\text { MI940 }\end{array}$ \\
\hline Is & 1903 & 59 & $1875-1946$ & $\begin{array}{l}1873-1947 \\
94 a\end{array}$ & FI908 & \\
\hline $\begin{array}{l}16 \\
17\end{array}$ & $\begin{array}{l}1901 \\
1912\end{array}$ & $\begin{array}{l}56 \\
49\end{array}$ & $\begin{array}{l}1875-1941 \\
1880-1957\end{array}$ & $1873-1963 \mathrm{ND}$ & $\begin{array}{l}\text { MIg02 } \\
\text { Fig1I }\end{array}$ & FI934; Mr936 \\
\hline $\begin{array}{l}18 \\
19\end{array}$ & $\begin{array}{l}1902 \\
1916\end{array}$ & $\begin{array}{l}58 \\
48\end{array}$ & $\begin{array}{c}1875-1923 \\
1882-1942 \\
94 a\end{array}$ & $\begin{array}{l}1869-1927 \\
1869-1928\end{array}$ & $\begin{array}{l}\text { FI909 } \\
\text { Mi920 }\end{array}$ & 年 \\
\hline 20 & $\begin{array}{l}1910 \\
1904\end{array}$ & $\begin{array}{l}54 \\
59\end{array}$ & $\begin{array}{l}1873 \\
1880-194 I \\
93 c .3\end{array}$ & $\begin{array}{c}1871-1926 \\
1879-1947 \\
94 a\end{array}$ & $\begin{array}{l}\text { Migo3 } \\
\text { Figos }\end{array}$ & Mr942; Fr944 \\
\hline $\begin{array}{l}22 \\
23 \\
24\end{array}$ & $\begin{array}{l}1929 \\
1928 \\
1905\end{array}$ & $\begin{array}{l}35 \\
33 \\
58\end{array}$ & $\begin{array}{l}1899 \\
1900-1930 \mathrm{ND} \\
1876-1961 \\
420.1\end{array}$ & $\begin{array}{l}1898 \\
1893-1960 \\
1880-1952\end{array}$ & $\begin{array}{l}\text { Mrg27 } \\
\text { Mr927 } \\
\text { Frgo2 }\end{array}$ & $\begin{array}{l}\text { Mr954; Fr957 } \\
\text { Mr96I }\end{array}$ \\
\hline 25 & 1905 & 51 & $1880-1923$ & IE & Fi917 & $\begin{array}{l}\text { Frg32; [Fr943; Fr943] } \\
\quad\left(\mathrm{M}_{3} ; \mathrm{Fo}\right)\end{array}$ \\
\hline 26 & 1904 & 60 & $\begin{array}{l}187 I-1947 \\
94 a\end{array}$ & $1871-1952$ & Migro & \\
\hline $\begin{array}{l}27 \\
28\end{array}$ & $\begin{array}{l}1901 \\
1902\end{array}$ & $\begin{array}{l}58 \\
59\end{array}$ & $\begin{array}{l}1872-1942 \mathrm{~W} \\
1865-1960\end{array}$ & $\begin{array}{l}1872-1942 \mathrm{~W} \\
1854-1938\end{array}$ & $\begin{array}{l}M I 895-1963 \\
422.2 \\
M r 909 ;(M 0)\end{array}$ & \\
\hline 29 & $\begin{array}{l}1902 \\
1926\end{array}$ & 59 & $\begin{array}{l}1865-1960 \\
420.1 \\
1897-1963\end{array}$ & $\begin{array}{l}1854-1938 \\
188 I-194 I\end{array}$ & Mig30 & FI951; Fro57 \\
\hline 30 & $\begin{array}{l}1926 \\
1904\end{array}$ & $\begin{array}{l}35 \\
58\end{array}$ & $\begin{array}{l}1897-1963 \\
1873-1907\end{array}$ & $\begin{array}{l}1881-1941 \\
94 a \\
1879-1954 \mathrm{ND}\end{array}$ & $\begin{array}{l}\text { Figoo; (Fo) (Pat. } \frac{1}{2} \text { sib m, 1913; } \\
\text { f, 1917-1961 f, 1915) }\end{array}$ & Fi929; Fig35 \\
\hline $\begin{array}{l}3 \mathbf{I} \\
\mathbf{3 2}\end{array}$ & $\begin{array}{l}1914 \\
\text { I904 }\end{array}$ & $\begin{array}{l}47 \\
58\end{array}$ & $\begin{array}{l}1883 \\
1875-1944\end{array}$ & $\begin{array}{l}1883 \\
1873-1959 \\
422.2\end{array}$ & $\begin{array}{l}\text { 33I } \\
\text { Migro-r933W } \\
\text { Migi3 }\end{array}$ & Mr946 \\
\hline 33 & I904 & 59 & $\begin{array}{c}I 873-1934 \\
93 b .3\end{array}$ & $1878-1959$ & Figro & \\
\hline \multicolumn{7}{|c|}{ Three-child families } \\
\hline $\begin{array}{l}34 \\
35\end{array}$ & $\begin{array}{l}\text { I9II } \\
\text { 1909 }\end{array}$ & $\begin{array}{l}52 \\
44\end{array}$ & $\begin{array}{l}\text { I 878-I93I } \\
\text { I } 880^{\star}\end{array}$ & $\underset{1880-1928}{\text { IE }}$ & $\begin{array}{l}\text { Migo3; Figo6 } \\
\text { Migo6-1962; Fig1 } \\
\quad 420.1\end{array}$ & $\begin{array}{l}\text { Fig42; Fr944; Fr946 } \\
\text { Fi948; Fr949 }\end{array}$ \\
\hline 36 & I904 & 58 & $1883-1951$ & $1875-1937$ & $\begin{array}{l}M 1903-1957 ; F_{1904} \\
\quad 420.1\end{array}$ & Mr937; Fi943 \\
\hline 37 & 1906 & 57 & $1877-1925$ & 1874-1947 & Fi902; Mig12; (FI5; Fi5) & $\begin{array}{l}\text { Mr929; Mr934; Mr940; } \\
\text { Mr948 }\end{array}$ \\
\hline $\begin{array}{l}38 \\
39\end{array}$ & $\begin{array}{l}1908 \\
1906\end{array}$ & $\begin{array}{l}44 \\
57\end{array}$ & $\begin{array}{l}1879-1963 \\
1885-1945\end{array}$ & $\begin{array}{l}1878-1960 N D \\
1878-1951 \\
332\end{array}$ & $\begin{array}{l}\text { Mr907; Fr912 } \\
\text { Fr909; FI912; (MI) }\end{array}$ & $\begin{array}{l}\text { Fig32; MI933; Fr937 } \\
\text { Fr945 }\end{array}$ \\
\hline 40 & I903 & 57 & $\begin{array}{l}1875-1956 \\
422.2\end{array}$ & $\begin{array}{l}1874-1946 \\
93 c .1\end{array}$ & Mi899; MrgI4-F965 SP & \\
\hline $\begin{array}{l}41 \\
42 \\
43 \\
44\end{array}$ & $\begin{array}{l}1905 \\
1908 \\
1915 \\
1910\end{array}$ & $\begin{array}{l}55 \\
51 \\
48 \\
53\end{array}$ & $\begin{array}{l}1871-1960 \\
1884-1950 \\
1881 \\
1888-1923\end{array}$ & $\begin{array}{l}1880-1939 \\
1881-1919 \\
1858-1932 \\
1885-1935 \\
97.1\end{array}$ & $\begin{array}{l}\text { Mr903-I962; Migro } \\
\text { Fr908; Mr914; (Mat. } \frac{1}{2} \text { sib m23) } \\
\text { Mr905; Mr927 } \\
\text { FI916; Fr92I-194I; (Mr, M5) }\end{array}$ & $\begin{array}{l}\text { Fr930; Fr932 } \\
\text { Mr938; Mrg42 } \\
\text { Mr939; Fr942; Fr946; } \\
\text { Mr940; Fr942; Fis } \\
\text { Frg55 }\end{array}$ \\
\hline 45 & 1913 & 49 & 1878 & $1880-1916$ & $\begin{array}{l}\text { Mr912; Mr916; (Mat. } \frac{1}{2} \text { sibs mI896; } \\
\text { fr900; mr904) }\end{array}$ & Fi942 \\
\hline 46 & 1929 & 34 & 1900 & $\begin{array}{c}1903-1960 \\
420.1\end{array}$ & Mi926; Fr927 & Fi955; Fr956; Fr958 \\
\hline $\begin{array}{l}47 \\
48 \\
49 \\
50\end{array}$ & $\begin{array}{c}1904 \\
1902 \\
1901-1964 \\
1906\end{array}$ & $\begin{array}{l}57 \\
57 \\
59 \\
57\end{array}$ & $\begin{array}{l}1869-1942 \mathrm{ND} \\
1886-1953 \\
1878-1949 \mathrm{ND} \\
1880-192^{\star} \\
\quad 94 a\end{array}$ & $\begin{array}{l}1850-1909 \\
1883-1961 \\
1876-1918 \\
1879\end{array}$ & $\begin{array}{l}\text { Mi898; Figo6; (Mat } \frac{1}{2} \text { sib migrs) } \\
\text { Fr905; Mr907 } \\
\text { Fr908-I947; Fr909 } \\
\text { Mr904; Mr914 }\end{array}$ & $\begin{array}{l}\text { Fr930; Fr932 } \\
\text { Fr927; Fr932; Fr940; (Mo) } \\
\text { Mr928; Mr932; Fr933; } \\
\text { Fr936; Fr945 }\end{array}$ \\
\hline
\end{tabular}


Appendix II-continued

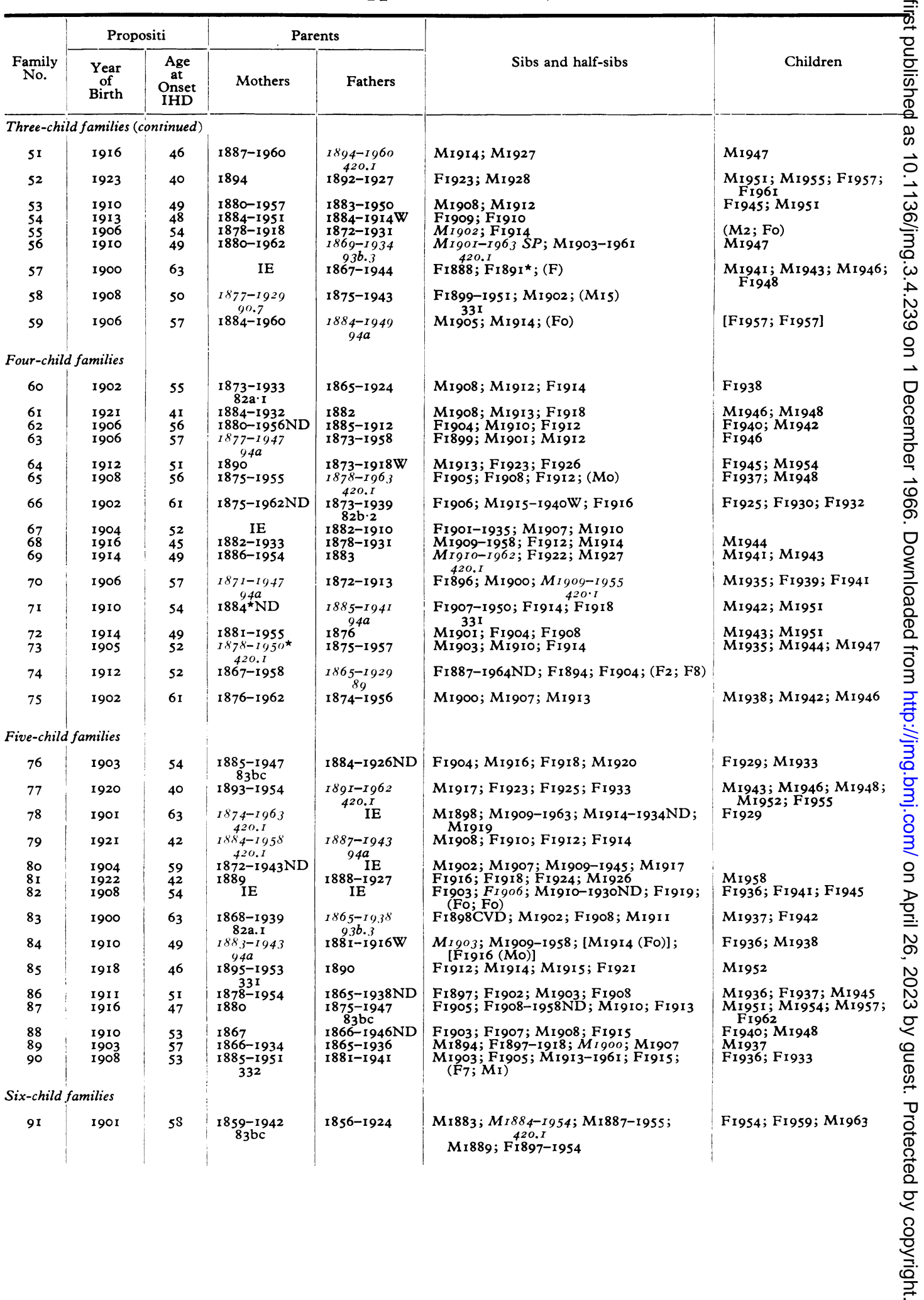


Appendix II-continued

\begin{tabular}{|c|c|c|c|c|c|c|}
\hline \multirow[b]{2}{*}{$\begin{array}{l}\text { Family } \\
\text { No. }\end{array}$} & \multicolumn{2}{|c|}{ Propositi } & \multicolumn{2}{|c|}{ Parents } & \multirow[b]{2}{*}{ Sibs and half-sibs } & \multirow[b]{2}{*}{ Children } \\
\hline & $\begin{array}{l}\text { Year } \\
\text { of } \\
\text { Birth }\end{array}$ & $\begin{array}{l}\text { Age } \\
\text { at } \\
\text { Onset } \\
\text { IHD }\end{array}$ & Mothers & Fathers & & \\
\hline \multicolumn{7}{|c|}{ Six-child families (continued) } \\
\hline 92 & I904 & 50 & 1874 & I 877-1944 & Mr898-1950; Migoo; Mr904; Mr9o9; & Mr934; Fr939 \\
\hline 93 & 1913 & so & $\begin{array}{c}1883-1951 \\
331\end{array}$ & $1883-1918$ & $\begin{array}{l}\text { MrgoI-1955; Mig03; Fr904; } \\
\quad 420.1 \\
\quad \text { Mi907-1940; MI910-I964SP }\end{array}$ & Fr934; Fr938 \\
\hline 94 & 1904 & 55 & 1879 & $\begin{array}{c}1873-1934 \\
94\end{array}$ & FI900; Mr902; Mr908; Fi910; FI914 & $\begin{array}{l}\text { Mr925; Fi930; Fr943; } \\
\quad \text { Fr945 }\end{array}$ \\
\hline 95 & 1918 & 46 & $1890-1958$ & $1891-1956$ & Mr910; Fr916; Mr920; Mr922; Mr923 & MI942; Mi946 \\
\hline 96 & I909 & 54 & $1876-1921$ & $\begin{array}{l}332 \\
1867-1937 \\
97.3\end{array}$ & $\underset{\text { FI918 }}{\text { Mrgo5-I959; Fr906; Fr907; Fr916; }}$ & FI94I \\
\hline 97 & 1914 & 49 & $\begin{array}{c}1874-1940 \\
83 \mathrm{bc}\end{array}$ & $1873-1927$ & $\begin{array}{l}\text { Fr897; Mr899; Fr905; Mr906-1939; } \\
\text { Mig10 }\end{array}$ & \\
\hline 98 & 1921 & 42 & 1887 & I888-I939ND & $\begin{array}{l}\text { Mrgo9; Frg12; Mrg18-1940W; Fr926; } \\
\text { Mr932 }\end{array}$ & $\left(\mathbf{M}_{3}\right)$ \\
\hline 99 & 1916 & 45 & $\begin{array}{c}1889-1957 \\
332\end{array}$ & $\begin{array}{l}I 886-1952 \\
420.1\end{array}$ & Fr914; Fr918; Mr920; Fr922; Fr924 & Mr946 \\
\hline 100 & 1912 & 46 & $1876-1949$ & $1876-1929$ & $\begin{array}{l}\text { Mig02-1951; Fig03; Fig05; Fig07; } \\
\text { 420.I } \\
\text { Mi910; MI9I4 }\end{array}$ & Mi938 \\
\hline \multicolumn{7}{|c|}{ Seven-child families } \\
\hline IOI & 1929 & 35 & $\begin{array}{c}1895-1939 \\
97 \cdot 1\end{array}$ & 1895 & $\begin{array}{c}\text { Migr6; Mig18; MI920-1964; Fig2I*; } \\
420.1\end{array}$ & Mi950; Fi953 \\
\hline 102 & 1906 & 52 & I 865-1944 & $1860-1929$ & $\begin{array}{l}\text { Fi884; M1890-1961; Fr899; Mr902; } \\
\text { Mrgo5-1963;[M1906 SP propositor] }\end{array}$ & \\
\hline 103 & I910 & 37 & 1868-1959ND & $1858-1921$ & MI890; FI894; MI894-I96I; FI899; & Mr940; Mr943 \\
\hline 104 & 1904 & 59 & 1880 & $1878-1951$ & $\begin{array}{l}\text { FI904; F1910;(F4; Mo) } \\
\text { MI896; MI901; FI908; FI916; Mr910; } \\
\text { Fr920;(F2) }\end{array}$ & Mr944; Mr948 \\
\hline 105 & 1907 & 57 & $1885-1961$ & $I 873-I 96 I$ & Mrgo4; Mrgo9; Mr914; Mr916; Mr927; & Mi950; Fi952 \\
\hline 106 & 1894 & 63 & I860-I94IND & $1855-1911$ & $\begin{array}{l}\text { Fi877; Fi880; F1883; Mr885; Mr891; } \\
\text { F1900-1960 }\end{array}$ & $\begin{array}{l}\text { MI914-1962; MI917; } \\
\quad 420.1 \\
\text { MI92I-I942; Frg23; } \\
\text { Mig25; Mr93I }\end{array}$ \\
\hline 107 & 1909 & 56 & 1883 & $\begin{array}{l}I 876-196 I^{\star} \\
\quad 420.1\end{array}$ & $\begin{array}{l}\text { Frgo9; Fi911; Mig13; Mig15; Fi916; } \\
\text { FI926-1954 }\end{array}$ & \\
\hline 108 & I9II & 52 & 1882 & 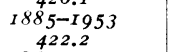 & $\begin{array}{l}\text { Figro; Mr918; Fi922; Fig24; Mr926; } \\
\text { FI928 }\end{array}$ & FI940; Fi948 \\
\hline 109 & 1919 & 45 & $\begin{array}{c}1880-1951 \\
422 \cdot 2\end{array}$ & $1869-1940$ & $\begin{array}{l}\text { Figo6; FI908; Fr910; FI913; MI914; } \\
\text { FI9I6 }\end{array}$ & $\left(\mathbf{M}_{3}\right)$ \\
\hline \multicolumn{7}{|c|}{ Eight-child families } \\
\hline I 10 & 1916 & 47 & 1889 & $\begin{array}{c}1883-I 94^{I} \\
94 a\end{array}$ & $\begin{array}{l}\text { Mr912; Mr919; Fr924; Mr932; Fr938; } \\
\text { Fr940; Fr942 }\end{array}$ & $\underset{\text { Frg53 }}{\text { Mrg46-1963; Mr948; }}$ \\
\hline II I & 1918 & 43 & I880-I 948 & 1879-1955 & $\begin{array}{l}\text { Frgo6; Mig07; Fig10; Figr2-1953ND } \\
\text { FI913; Fi916; [F1918; propositor] }\end{array}$ & FI945; FI948 \\
\hline 112 & 1909 & 52 & $1877-1948$ & 1878 & $\begin{array}{l}\text { Mrg0I; Frg02; MI904; FI906; Mr907; } \\
\text { FI908; FI91I }\end{array}$ & $\begin{array}{l}\text { Mr933; Mr935; Mr937; } \\
\text { Mr939; Mr941; Fr943 }\end{array}$ \\
\hline 113 & $1899-1963$ & 52 & $1870-1945$ & I869-I939ND & $\begin{array}{l}\text { Mr894; Mr897-1917W; Fr9o1; } \\
\text { Mr903-1923; Fr905; Mr907; Mr909 }\end{array}$ & Frg27 \\
\hline 114 & I9II & 48 & 1889 & $\begin{array}{l}1885-1936 \\
97.1\end{array}$ & 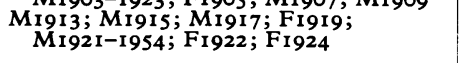 & FI938; MI944 \\
\hline \multicolumn{7}{|c|}{ Nine-child families } \\
\hline $\begin{array}{l}115 \\
116\end{array}$ & $\begin{array}{l}1902 \\
1909\end{array}$ & $\begin{array}{l}6 I \\
49\end{array}$ & $\begin{array}{l}1866-19.38 \\
9.36 .2 \\
1868-1915\end{array}$ & $\begin{array}{l}\text { I 866-I } 934 \\
\text { I862-I929 }\end{array}$ & 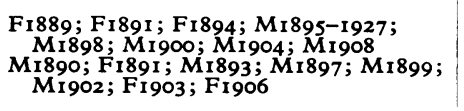 & $F_{1938}$ \\
\hline \multicolumn{7}{|c|}{ Ten-child families } \\
\hline 117 & 1920 & 42 & 1879-1958 & I 878-I 945 & 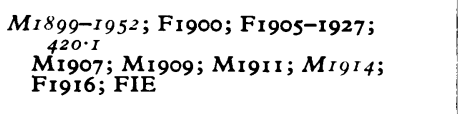 & M1947; Mr949 \\
\hline
\end{tabular}


Appendix II-continued

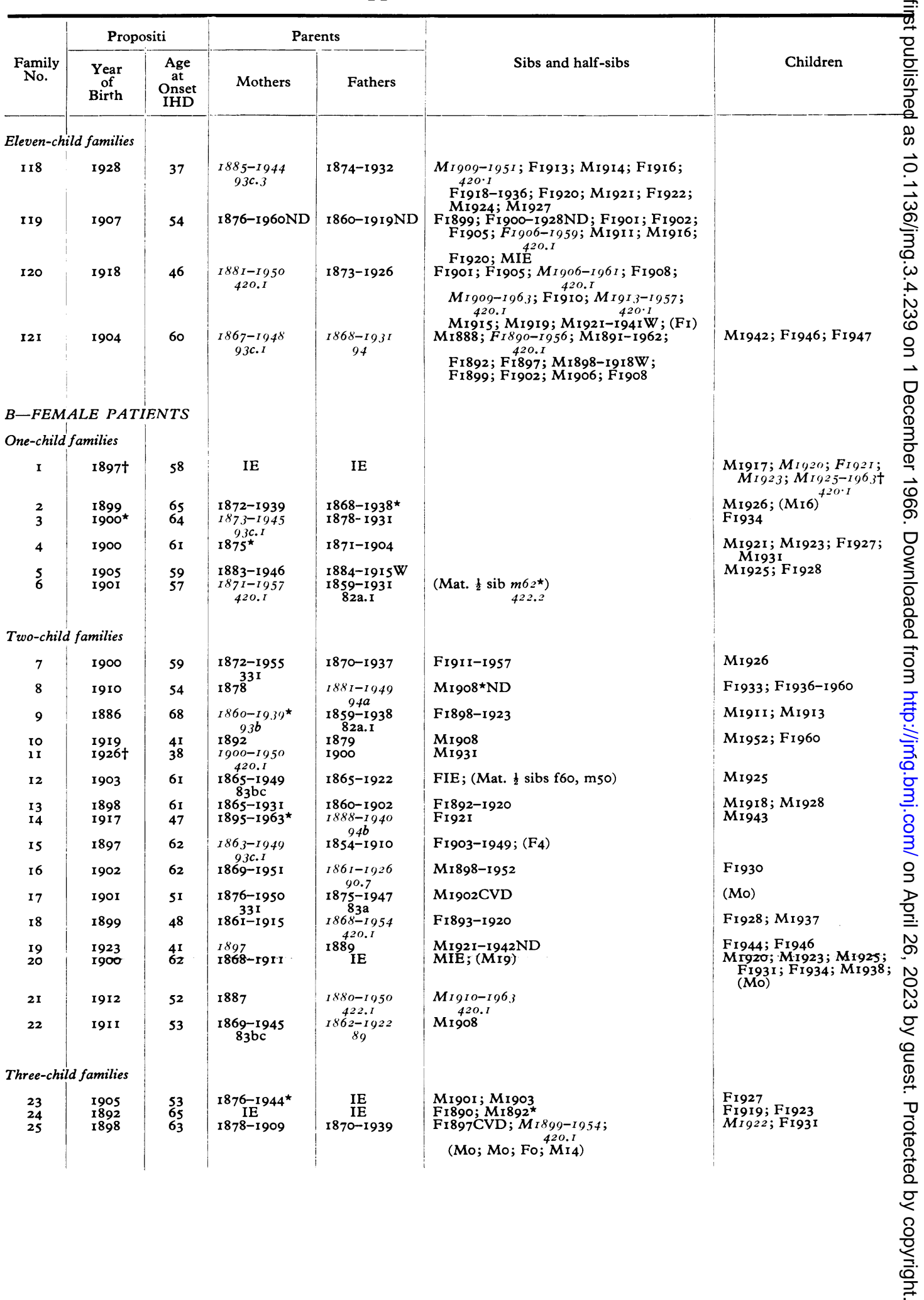


Appendix II-continued

\begin{tabular}{|c|c|c|c|c|c|c|}
\hline \multirow[b]{2}{*}{$\begin{array}{l}\text { Family } \\
\text { No. }\end{array}$} & \multicolumn{2}{|c|}{ Propositi } & \multicolumn{2}{|c|}{ Parents } & \multirow[b]{2}{*}{ Sibs and half-sibs } & \multirow[b]{2}{*}{ Children } \\
\hline & $\begin{array}{c}\text { Year } \\
\text { of } \\
\text { Birth }\end{array}$ & $\begin{array}{l}\text { Age } \\
\text { at } \\
\text { Onset } \\
\text { IHD }\end{array}$ & Mothers & Fathers & & \\
\hline \multicolumn{7}{|c|}{ Three-child families (continued) } \\
\hline $\begin{array}{l}\mathbf{2 6} \\
\mathbf{2 7}\end{array}$ & $\begin{array}{l}1899 \\
1902\end{array}$ & $\begin{array}{l}55 \\
62\end{array}$ & $\begin{array}{l}1877-1933 \\
1868-193 I^{\star}\end{array}$ & $\begin{array}{l}1876-1930 \\
1857-1910 \\
79 b\end{array}$ & $\begin{array}{l}\text { Migo4; Mrgro-r940W; (MI; Fo) } \\
\text { Mr896-I929; Fr902-I932 }\end{array}$ & Mi923; Fig25 \\
\hline $\begin{array}{l}28 \\
29\end{array}$ & $\begin{array}{l}1913 \\
1925\end{array}$ & $\begin{array}{l}51 \\
39\end{array}$ & $\begin{array}{l}1887-1950^{\star} \\
1900\end{array}$ & $\begin{array}{c}1879-1949 \\
1880-1929 \\
90.7\end{array}$ & $\begin{array}{l}\text { Mig05; Mrgio-1942 } \\
\text { Mig21; Mig23 }\end{array}$ & \\
\hline 30 & 1896 & 54 & $\begin{array}{l}1865-194^{8} \\
9.3 \mathrm{c.I}\end{array}$ & $\begin{array}{c}1869-1947 \\
9.3 \mathrm{c} .3\end{array}$ & FI897-I957; FI908 & $\begin{array}{l}\text { Figr6; Mr923; Fi928; } \\
\text { MI933; MI937 }\end{array}$ \\
\hline 31 & I9II & 41 & 1882 & $1878-1917$ & $\begin{array}{l}\text { MI905-1959; Fig09; (FI9) } \\
\text { 420.I }\end{array}$ & Mr937 \\
\hline 32 & 1905 & 56 & I $881-1947$ & I879-1954 & $\begin{array}{l}\text { Migit-I962; Mi920 } \\
\quad+20.1\end{array}$ & \\
\hline 33 & 1909 & 55 & I 882-1935 & $\begin{array}{c}I 882-1955 \\
420 . I\end{array}$ & FI904; MI9I3 & \\
\hline 34 & I92I & 36 & I 890 & $\begin{array}{c}1889-1947 \\
93 \mathrm{c} .3\end{array}$ & MI91I; Fig26 & FI944 \\
\hline 35 & I9I I & 53 & $\begin{array}{l}1893-1956 \\
332\end{array}$ & IE & FI9I3; MI9I4 & FI934; FI948 \\
\hline $\begin{array}{l}36 \\
37\end{array}$ & $\begin{array}{l}1906 \\
1902\end{array}$ & $\begin{array}{l}58 \\
59\end{array}$ & $\begin{array}{l}1879-1918 \\
1866-1944 \\
93 c .3\end{array}$ & $\begin{array}{l}\text { IE } \\
1871-1955 \\
332\end{array}$ & $\begin{array}{l}\text { Mi908; Fi910 } \\
\text { Mr899; Mi916;(F6*) }\end{array}$ & $\begin{array}{l}\text { Mr93I } \\
\text { FI928; MI940 }\end{array}$ \\
\hline $\begin{array}{l}38 \\
39 \\
40\end{array}$ & $\begin{array}{l}\text { I } 901 \\
1905 \\
\text { I } 903\end{array}$ & $\begin{array}{l}63 \\
59 \\
61\end{array}$ & $\begin{array}{l}1866-1918 \\
1882-1930 \\
1870-1962^{\star} \\
332\end{array}$ & $\begin{array}{c}1862-1925 \\
1878-1930 \\
1869-1908 \\
B_{3}\end{array}$ & 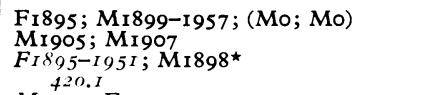 & $\begin{array}{l}\text { Mr930; Mr933 } \\
\text { Mi936; Mr938; Fr944 }\end{array}$ \\
\hline $4 I$ & I905t & 59 & $\begin{array}{c}1884-1941 \\
94 a\end{array}$ & I 879-1944 & MIgog; Fig22 & \\
\hline \multicolumn{7}{|c|}{ Four-child families } \\
\hline 42 & 1904 & 58 & $\underset{\substack{1874 \\
93}}{ }$ & $1869-1929$ & Mr899; Mr909; Fi916; (MI8W) & Mr932; FI937 \\
\hline $\begin{array}{l}43 \\
44\end{array}$ & $\begin{array}{l}1897^{\star} \\
1898\end{array}$ & $\begin{array}{l}67 \\
57\end{array}$ & $\begin{array}{c}1870 \\
1866-1929 \\
90.7\end{array}$ & $\begin{array}{l}1870-1923 \\
1865-1909\end{array}$ & $\begin{array}{l}\text { Mrgoo; Mig02; Fr907 } \\
\text { MI895-I95I; FI905; Mr906 } \\
\text { 420.I }\end{array}$ & Migis \\
\hline $\begin{array}{l}45 \\
46\end{array}$ & $\begin{array}{l}1916 \\
1909\end{array}$ & $\begin{array}{l}48 \\
50\end{array}$ & $\begin{array}{l}1889-1962 \\
420.1 \\
\text { IE }\end{array}$ & $\begin{array}{l}1889-1938 \\
82 a . I \\
1878-193 I^{\star}\end{array}$ & $\begin{array}{l}\text { MI9I4-I96o; Fig20; Fig29 } \\
\text { 420.I } \\
\text { FI904; MI905-I963; Fr906 }\end{array}$ & $\begin{array}{l}\text { Mr933; Fr936; Fr952; } \\
\text { FI954 }\end{array}$ \\
\hline 47 & I903 & 56 & $\begin{array}{c}1860-19.54 \\
422.2\end{array}$ & $1872-1956$ & FI896; Mr898; MI90I; $\left(\mathrm{FI}_{1} 7^{\star}\right)$ & \\
\hline 48 & I912 & 49 & $1890-1954^{\star}$ & $\begin{array}{c}I 886-1963 \\
420.1\end{array}$ & Mi913; Mr9i5; Fr924; (Mr4) & Mr947 \\
\hline 49 & I9I 8 & 45 & $\begin{array}{l}1893-1955 \\
420.1\end{array}$ & I895-1964 & Mi917; Mr920; Fi923 & Fi945 \\
\hline 50 & 1908 & 54 & $\begin{array}{c}1876-1953 \\
422.1\end{array}$ & $1874-1935$ & Mi902; Fig03; Mr905-I936 & \\
\hline 51 & $\begin{array}{l}\text { I897t } \\
\text { I9I I }\end{array}$ & $\begin{array}{l}57 \\
53\end{array}$ & $\begin{array}{l}1873-19+2 \\
93 c .3 \\
1873-1947\end{array}$ & $\begin{array}{l}\text { 1871-1944t } \\
\text { I869-1914 }\end{array}$ & $\begin{array}{l}F_{I 900-I 960 \dagger ;} \text { Mig02†; FI9It } \\
\text { 420.I } \\
\text { MI900; FI904; FIE }\end{array}$ & $\begin{array}{l}\text { Fi923; Fig24t; Fi926; } \\
\text { Mi931; Fi939 }\end{array}$ \\
\hline 53 & 1907 & 57 & $\begin{array}{c}93 c .3 \\
1872-196 \mathrm{I} \\
33 \mathrm{I}\end{array}$ & $1866-1916$ & FI9Io; FI9I4; FI9I8; (M7) & \\
\hline 54 & I9I I & 53 & $1882-1962$ & $1880-1925$ & Mi909; Migis; Figi8 & FI944; Mr949 \\
\hline 55 & $\begin{array}{l}1903 \\
1913\end{array}$ & $\begin{array}{l}54 \\
50\end{array}$ & $\begin{array}{l}1878-1938 \\
1891-1954 \\
331\end{array}$ & $\begin{array}{l}\text { I874-1956 } \\
\text { I 887-r } 924\end{array}$ & $\begin{array}{l}\text { Migoo; Migo5; Migo7-1949; (Mo) } \\
\text { Figo8; Mr9io; Migr2; (Mig) }\end{array}$ & MI93I \\
\hline \multicolumn{7}{|c|}{ Five-child families } \\
\hline $\begin{array}{l}57 \\
58\end{array}$ & $\begin{array}{l}\text { 1910* } \\
1903\end{array}$ & $\begin{array}{r}53 \\
61 \\
\end{array}$ & I 886 & $\begin{array}{c}1884-1960 \\
1852-1927 \\
90.9\end{array}$ & 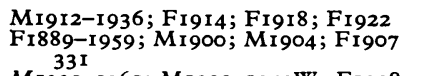 & Mr942 \\
\hline 59 & 1905 & 56 & $\begin{array}{l}1877-19.36 \\
\quad+20.1\end{array}$ & $\begin{array}{l}1875-1950 \\
420.1\end{array}$ & $\begin{array}{l}\text { MI90I-I96I; Mig02-1942W; Figo8; } \\
\text { 420.I } \\
\text { MI915; (Pat. } \frac{1}{2} \text { sib mi948) }\end{array}$ & Mr927; Mr932; Fr940 \\
\hline 60 & 1900 & 60 & 1866-19r5 & $\begin{array}{l}1863-1932 \\
82 a \cdot I\end{array}$ & Mr897; Mr899CVD; Fr913; Fr923 & \\
\hline 61 & 1920 & 44 & I886-I950 & $\begin{array}{c}1886-1952 \\
332\end{array}$ & MI907-I958; Fi913; Mr924*; Fr930 & Fr940 \\
\hline 62 & $1903^{\star}$ & 61 & $\begin{array}{l}1874-1942^{\star} \\
83 a^{\star}\end{array}$ & $1874-1942$ & 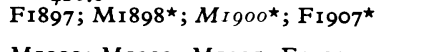 & Mr926; Mr929 \\
\hline $\begin{array}{l}63 \\
64\end{array}$ & $\begin{array}{l}1899 \\
1921\end{array}$ & $\begin{array}{l}62 \\
41\end{array}$ & $\begin{array}{l}1884^{-1954} \\
1894\end{array}$ & $\begin{array}{l}1877-1930 \\
1889-1947 \\
\quad 94 a\end{array}$ & $\begin{array}{l}\text { Mrgoo; Mr903; Mrgo5; Fr909 } \\
\text { Mr914; Mr916; Mr926; FI927-1962 }\end{array}$ & Fr926; Mr933 \\
\hline
\end{tabular}


Appendix I-continued

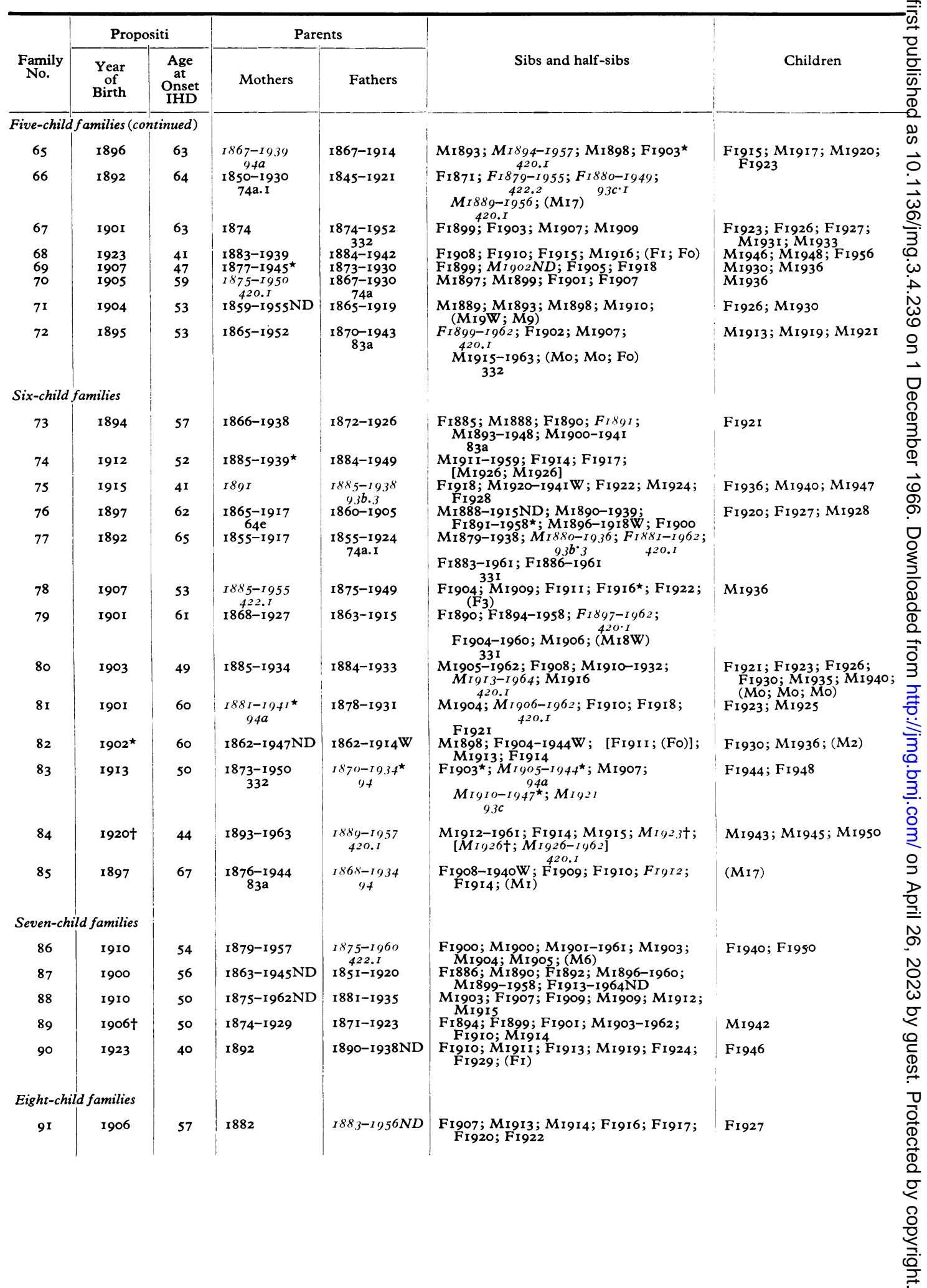


Appendix II-continued

\begin{tabular}{|c|c|c|c|c|c|c|}
\hline \multirow[b]{2}{*}{$\begin{array}{l}\text { Family } \\
\text { No. }\end{array}$} & \multicolumn{2}{|c|}{ Propositi } & \multicolumn{2}{|c|}{ Parents } & \multirow[b]{2}{*}{ Sibs and half-sibs } & \multirow[b]{2}{*}{ Children } \\
\hline & $\begin{array}{c}\text { Year } \\
\text { of } \\
\text { Birth }\end{array}$ & $\begin{array}{l}\text { Age } \\
\text { at } \\
\text { Onset } \\
\text { IHD }\end{array}$ & Mothers & Fathers & & \\
\hline \multicolumn{7}{|c|}{ Eight-child families (continued) } \\
\hline 92 & 1895 & 60 & $1871-1940$ & $\begin{array}{l}1 \times 6 \times-1979 \\
\quad 9.3 c .1\end{array}$ & $\begin{array}{l}\text { FI894; FI } 897 ; \text { MI899; MI90I; FI903; } \\
\quad \text { FI904; MI906 }\end{array}$ & Fr922; Mr932 \\
\hline \multicolumn{7}{|c|}{ Nine-child families } \\
\hline 93 & 1895 & 63 & $\begin{array}{l}1865-1945 \\
83 \mathrm{bc}\end{array}$ & 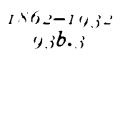 & 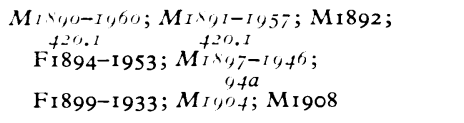 & Mi928; Fi931 \\
\hline \multicolumn{7}{|c|}{ Ten-child families } \\
\hline 94 & I914t & 50 & $1877-1959$ & $1874-1950$ & 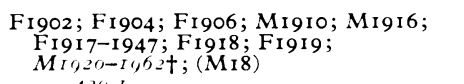 & \\
\hline 95 & I 901 & 62 & 1873-I950ND & 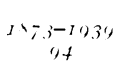 & 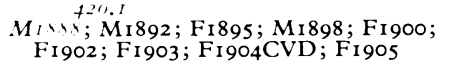 & Fi 938 \\
\hline \multicolumn{7}{|c|}{ Eleren-child families } \\
\hline 96 & 1910 & 53 & $1887-1957^{\star}$ & $\begin{array}{l}1885-1937 \\
\quad 82 \mathrm{a} . \mathrm{I}\end{array}$ & 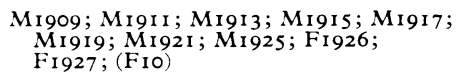 & Fr 933 \\
\hline
\end{tabular}

